Colloid and Interface Science

Elsevier Editorial System(tm) for Journal of

Manuscript Draft

Manuscript Number: JCIS-19-6097R1

Title: Self-Assembled Micro-stripe Patterning of Sessile Polymeric Nanofluid Droplets

Article Type: Full length article

Section/Category: A. Colloidal Materials and Nanomaterials

Keywords: nanofluids; nanoparticles; microfluidics; self-assembly.

Corresponding Author: Professor Mohan Edirisinghe, DSC

Corresponding Author's Institution: UCL

First Author: Merve Gultekinoglu

Order of Authors: Merve Gultekinoglu; Xinyue Jiang; Cem Bayram; Han Wu; Kezban Ulubayram; Mohan Edirisinghe, DSC

Abstract: When sessile nanofluid droplets evaporate, solid nanoparticles can be organized in a wide variety of patterns on the substrate. The composition of the nanofluid, internal flow type of droplet and the rate of drying affect drop geometry, and the final pattern. Using poly(lacticco-glycolic acid)-block-poly (ethylene glycol) (PLGA-b-PEG) as the example, we produced micro-stripe patterning from nanoparticles by drying of sessile fluid droplets. We investigated the nanoparticle properties and flow dynamics to clarify their effects on the patterning. Nanoparticles were prepared by hydrodynamic flow focusing using a T-junction microfluidic device with high production efficiency and the ability to generate an extremely narrow size distribution. PLGA-b-PEG was prepared as oil phase in acetonitrile and water/oil flow rate was changed from 1 to 3 at constant oil phase flow rate $(50 \mu \mathrm{L} / \mathrm{min})$. Then, nanofluid was collected on the surface as sessile droplets within acetonitrile/water binary dispersed phase. Depending on size, charge and size-distribution, the nanoparticles deposited on the surface exhibited various patterns. Dynamic Light and $X$-ray Scattering measurements showed that, approximately $100 \mathrm{~nm}$ particles with relatively low PDI (0.04) were produced for the first time in surfactant free conditions in a microfluidic device and they generated self-assembled ordered patterns, which are regulated by the type of internal flow in the sessile nanofluid droplet during sequential evaporation of acetonitrile and water. 
Professor Arthur T. Hubbard, Ph.D.

Co-Editor

Journal of Colloid and Interface Science

Manuscript No: JCIS-19-6097

Dear Professor Hubbard,

We thank the reviewers and you immensely.

Enclosed please find our response to reviewer comments. The comments are answered one by one and required changes are highlighted in the revised manuscript. We hope the manuscript is in order now and we look forward to acceptance. We have also emailed you separately regarding a possible front cover.

With my kindest regards,

Yours sincerely

Professor Mohan Edirisinghe (Corresponding author) 


\section{RESPONSE TO REVIEWERS}

\section{Reviewer 1:}

Comments:

In this work, the authors have synthesized PLGA-b-PEG nanoparticles by evaporating droplets formed using a T-junction microfluidic device. Authors have characterized the resultant droplets using various analytical techniques such as DLS, SEM, and SAXS after drying of the droplet. The final particles were found to be $100 \mathrm{~nm}$ in size with very low PDI in the surfactant-free environment. Overall, this is a nice piece of work that can be published after minor revisions noted below.

Question 1: 1) P 6 L10: It should be 'figure 1', not 'figure 8'.

Answer 1: Thank you for your attention. In the Page 6, Line 10, the related Figure number "Figure 8" was corrected in the manuscript as "Figure 1" and highlighted.

Question 2: Is it possible to decrease the size of nanoparticles if solution concentration is increased to more than $10 \%$ ?

Answer 2: During the study, PLGA-b-PEG polymer solution was prepared at 2.5, 5 and $10 \%(\mathrm{w} / \mathrm{v})$ concentrations in acetonitrile and the solutions used directly. $10 \%$ was successfully used in the T-junction. It was not possible to process any higher concentration as their higher viscosity caused frequent blockage. This explanation is in the manuscript.

Question 3: Currently, droplets have been dried under ambient conditions. Will there be any effect of drying temperature?

Answer 3: PLGA-b-PEG polymer has a glass transition temperature $(\mathrm{Tg})$ of $\sim 41-43$ ${ }^{\circ} \mathrm{C}$, and therefore droplet drying procedure was carried out starting from ambient conditions up to $40{ }^{\circ} \mathrm{C}$. However, the studies were continued at room temperature since increasing temperature did not have shown significant effect on the obtained patterns. 

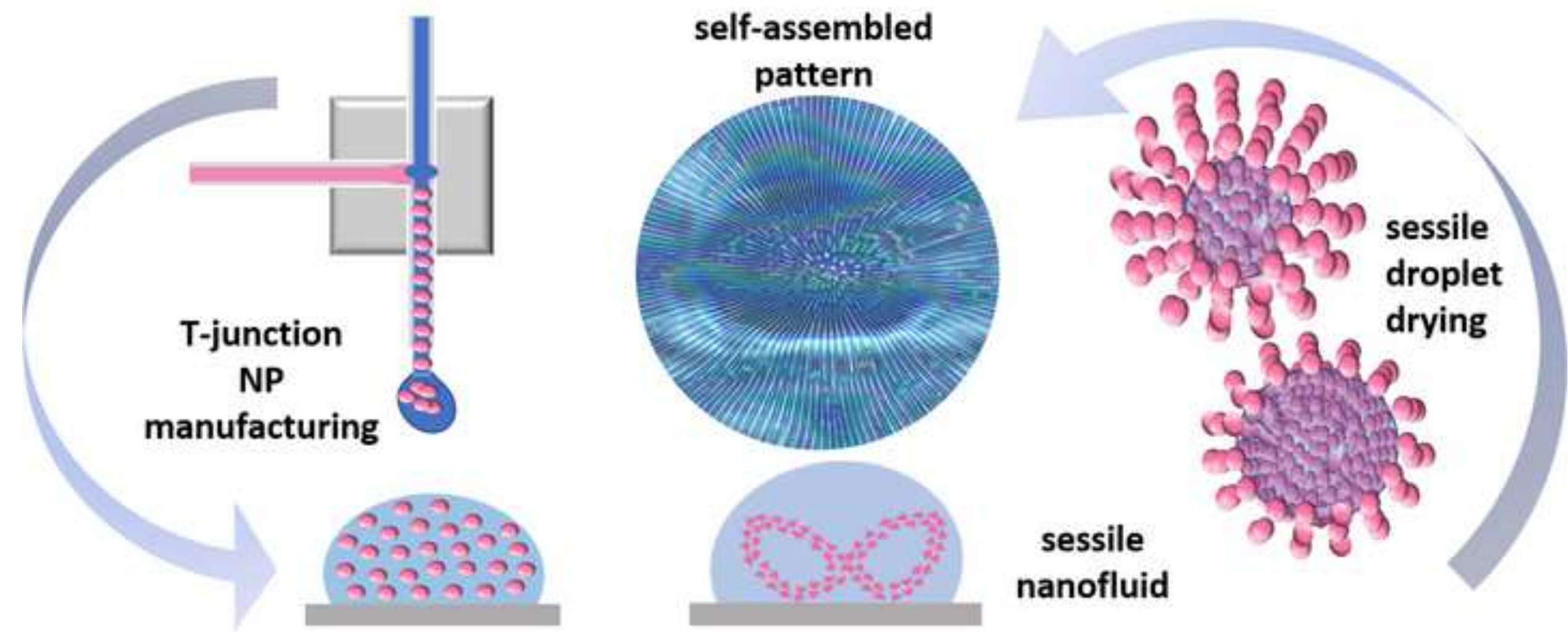


\title{
Self-Assembled Micro-stripe Patterning of Sessile Polymeric Nanofluid Droplets
}

 \\ Kezban Ulubayram ${ }^{1,2,6,7}$, Mohan Edirisinghe ${ }^{3 *}$
}

${ }^{1}$ Department of Basic Pharmaceutical Sciences, Faculty of Pharmacy, Hacettepe University, Turkey

${ }^{2}$ Bioengineering Division, Institute for Graduate Studies in Science \& Engineering, Hacettepe University, Turkey

${ }^{3}$ Department of Mechanical Engineering, University College London (UCL), London WC1E 7JE, UK

${ }^{4}$ Advanced Technologies Application and Research Center, Hacettepe University, Ankara, Turkey

${ }^{5}$ Department of Chemical Engineering, University College London (UCL), London, WC1E 7JE, UK

${ }^{6}$ Nanotechnology \& Nanomedicine Division, Institute for Graduate Studies in Science \& Engineering, Hacettepe University, Turkey

${ }^{7}$ Polymer Science and Technology Division, Institute for Graduate Studies in Science \& Engineering, Hacettepe University, Turkey

* Corresponding author ME- m.edirisinghe@ucl.ac.uk 


\section{Abstract}

When sessile nanofluid droplets evaporate, solid nanoparticles can be organized in a wide variety of patterns on the substrate. The composition of the nanofluid, internal flow type of droplet and the rate of drying affect drop geometry, and the final pattern. Using poly(lactic-co-glycolic acid)-block-poly(ethylene glycol)(PLGA-b-PEG) as the example, we produced micro-stripe patterning from nanoparticles by drying of sessile fluid droplets. We investigated the nanoparticle properties and flow dynamics to clarify their effects on the patterning. Nanoparticles were prepared by hydrodynamic flow focusing using a T-junction microfluidic device with high production efficiency and the ability to generate an extremely narrow size distribution. PLGA-b-PEG was prepared as oil phase in acetonitrile and water/oil flow rate was changed from 1 to 3 at constant oil phase flow rate $(50 \mu \mathrm{L} / \mathrm{min})$. Then, nanofluid was collected on the surface as sessile droplets within acetonitrile/water binary dispersed phase. Depending on size, charge and size-distribution, the nanoparticles deposited on the surface exhibited various patterns. Dynamic Light and X-ray Scattering measurements showed that, approximately $100 \mathrm{~nm}$ particles with relatively low PDI (0.04) were produced for the first time in surfactant free conditions in a microfluidic device and they generated self-assembled ordered patterns, which are regulated by the type of internal flow in the sessile nanofluid droplet during sequential evaporation of acetonitrile and water.

Keywords: Nanofluid, hydrodynamic flow focusing, coffee-ring, self-assembly. 


\section{Introduction}

Nanofluid is basically defined as a colloidal suspension that contains nano-sized particles (nanoparticle-fluid suspensions). The suspended particles can be composed of metal, carbon or polymeric materials with various forms such as spherical nanoparticles ${ }^{1}$, carbon nanotubes ${ }^{2}$, nanowires ${ }^{3}$, nanofibers ${ }^{4}$ etc. Nanofluids generate uniform/non-uniform patterns when a sessile drop of suspension dries on a collecting layer. The sessile drops of nanofluids have an internal flow during the drying period and the pattern structure depends on this internal flow type. Moreover, nanoparticle size, shape, polydispersity, charge and material type directly affects the flow type and the pattern structure ${ }^{5-6}$. In addition, nanofluids have different evaporation, flow and spreading dynamics in contrast to pure solutions. In a sessile droplet of nanofluid, there are two main internal flow types, which are defined as Marangoni flow and Capillary flow.

A drying liquid droplet containing suspended solid particles leaves a ring-shaped trace along the boundary. This phenomenon is called coffee ring structure and can be encountered in daily life, such as stains left from coffee spills ${ }^{7}$. The scientific explanation of the coffee ring formation is based on fluid dynamics reported by Deegan et al. ${ }^{8}$ for the first time. The coffee ring formation is mainly caused by the solvent evaporation at the edge of the droplets. The edges are refilled with solvent redrawn from the centre and the solute carried by the flow also deposits at the edge, leaving a ring structure formation caused by particle deposition. However, in clean droplet interfaces without any surfactants, thermal Marangoni flow, which is caused by the evaporation, is also dominant in coffee ring structure formation on the centre of the droplet, rather than at the edges ${ }^{9}$. Basically, capillary flow induces uniform 
coffee-ring deposition, concentric rings and central deposition are induced by

Marangoni flow. In addition to elucidation of both, Sangani et al. ${ }^{10}$ reported that contact line of the coffee ring also depends on suspended particle size and concentration. These authors stated that the particles drawn to the edge may prevent the drop edge from receding during evaporation and cause more deposit formation. Revealing the transport phenomena of nanoparticles in evaporating droplets will lead to essential applications in lithography ${ }^{11}$, nanoparticle based coating $^{12}$, self-assembled patterns ${ }^{13}$ and bioseparation systems ${ }^{14}$.

For producing nanofluids which contains nanoparticles with small and narrow size distribution, homogeneous reaction and mixing conditions are crucial. Commonly, diminution of the device linear dimension, increase of temperature and concentration gradients are vital. Whitesides ${ }^{15}$ also stated that the microfluidic technologies have a pivotal role in monodisperse bubble, particle or droplet production due to controllable multiphase flow capabilities. Since microfluidic devices can supply accurate manipulation of mixing, residence time and reaction temperature, it has evolved into the most promising approaches to construct an effective mixing environment for nanoparticle preparation.

In contrast to conventional nanofluidic production methods, in the present study a precisely tuneable flow-focusing nanoprecipitation channel has been successfully created through a ready $\mathrm{T}$-junction micro-channel. In the literature, microfluidic technology was adapted for nanoparticle synthesis with microchip fluidic systems and there are several examples ${ }^{16-20}$. For instance, Valencia et al. ${ }^{16}$ performed nanoparticle synthesis with the aid of flow focusing in several microfluidic studies. In their work, polydimethylsiloxane (PDMS) micro-moulded channels were prepared and the main stream enabled flow of a polymeric solution, then they fed water phase from 
two parallel inlets and created the flow focusing to precipitate the nanoparticles. In our case, we chose the main flow stream as water and fed the polymer solution via lateral inlet of a $\mathrm{T}$-junction with a $90^{\circ}$ angle to the water stream. The polymer solution cut the water stream on the junction point of microfluidic device and feeding the polymer solution with controlled speed and concentration removes the need for surfactant in the synthesis. This is the very first design to make good use of laminar flow with low Reynolds number inside a microfluidic T-junction to produce a soft boundary co-axial nanoscale channel for one-step and surfactant free synthesis of nanoparticles. In contrast to traditional microfluidics, nanoparticles have been directly formed along the soft boundary co-axial channel, instead of forming microbubbles by immiscible solvents and burst to produce nanoparticles from their shells ${ }^{21}$. In this paper, we aimed to prepare self-assembled layers from concentrated nanofluids using PLGA-b-PEG polymeric nanoparticles with extremely low polydispersity index as an example, where layers are produced with the aid of our Tjunction microfluidic nanoparticle generation technique. For this purpose, PLGA-bPEG nanoparticles were synthesized in a $\mathrm{T}$-junction microfluidic device via hydrodynamic flow focusing. Then, self-assembled and highly ordered micro-stripe patterns were obtained according to the physicochemical characteristics of the nanofluids. Obtained pattern structures were characterized for size, charge, morphology and orientation. The effect of deposition parameters on the pattern structure is discussed in detail.

\section{Experimental section}

Materials: PLGA-b-PEG, 50:50, Resomer RGP d50105, d50155 (Mw=50000 g/mol) (diblock, 10\% and 15\% PEG) was obtained from Boehringer Ingelheim Pharma GmbH\&Co Ingelheim, Germany. Acetonitrile (anhydrous, 99.8\%) was obtained from 
Sigma-Aldrich, Germany. Polydimethylsiloxane (PDMS) microfluidic device (Tjunction) set up was fabricated by CNC machine in the University College London (UCL) Mechanical Engineering workshop, UK.

PLGA-b-PEG Polymer Solution Preparation and Characterization: PLGA-b-PEG polymer solution was prepared at 2.5, 5 and $10 \%(\mathrm{w} / \mathrm{v})$ concentrations in acetonitrile. Solution were magnetically stirred for $\sim 2$ hours prior to usage. It was not possible to process any higher concentration as their higher viscosity caused frequent blockage in the T-junction.

Nanofluid Production via T-junction Microfluidic Device: Microfluidic T-junction device assembly is described in detail in our previous work ${ }^{22-23}$. T-junction microfluidic device and nanofluid production by hydrodynamic flow focusing inside the junction are schematically represented in the Figure 1. Herein, two miscible phases were both fed by syringe pumps (Harvard Apparatus Ltd., Edenbridge UK) through FEP (Fluorinated Ethylene Polypropylene) tubing (both inner diameters at $200 \mu \mathrm{m}$ ) into a polydimethylsiloxane (PDMS) block. Another FEP capillary tubing (inner diameter $=200 \mu \mathrm{m}$ ) was fixed into the PDMS block as an outlet of the Tjunction. Water was fed into the junction from $y$-axis and oil phase (PLGA-b-PEG dissolved in acetonitrile) was fed from $x$-axis (Figure 1A). While the oil phase flow rate was kept constant at $50 \mu \mathrm{L} / \mathrm{min}$ throughout the study, the water flow rates of 50 $\mu \mathrm{L} / \mathrm{min}, 100 \mu \mathrm{L} / \mathrm{min}$ and $150 \mu \mathrm{L} / \mathrm{min}$ were evaluated. Owing to the miscibility of solvents, acetonitrile and water, an overlapping zone was created in the middle of outlet tubing. A phantom 7.3 high speed camera with a maximum resolution of $800 \times 600$ pixels at up to $4800 \mathrm{fps}$ giving a recording duration of 1.2 seconds (Vision Research Ltd., UK) was utilized to document formation of the hydrodynamic flow focusing inside the T-junction channel. This overlapping zone also formed a soft 
boundary co-axial nanoscale channel inside the outlet tubing, where PLGA-b-PEG nanoparticles were later precipitated. Precipitated nanoparticles were collected using glass slides from the end of outlet tubing of the microfluidic T-junction as shown in Figure 1B. Sessile nanofluid droplet drying and the micro-stripe pattern formation are shown by schematic representation (Figure 1C) and the optical microscope images (Figure 1D). A video showing the process in action is given in Supplementary Information. PLGA-b-PEG polymer has a glass transition temperature $(\mathrm{Tg})$ of $\sim 41-43$ ${ }^{\circ} \mathrm{C}$, and therefore droplet drying procedure was carried out starting from ambient conditions up to $40{ }^{\circ} \mathrm{C}$. However, the studies were continued at room temperature since increasing temperature did not have shown significant effect on the obtained patterns.

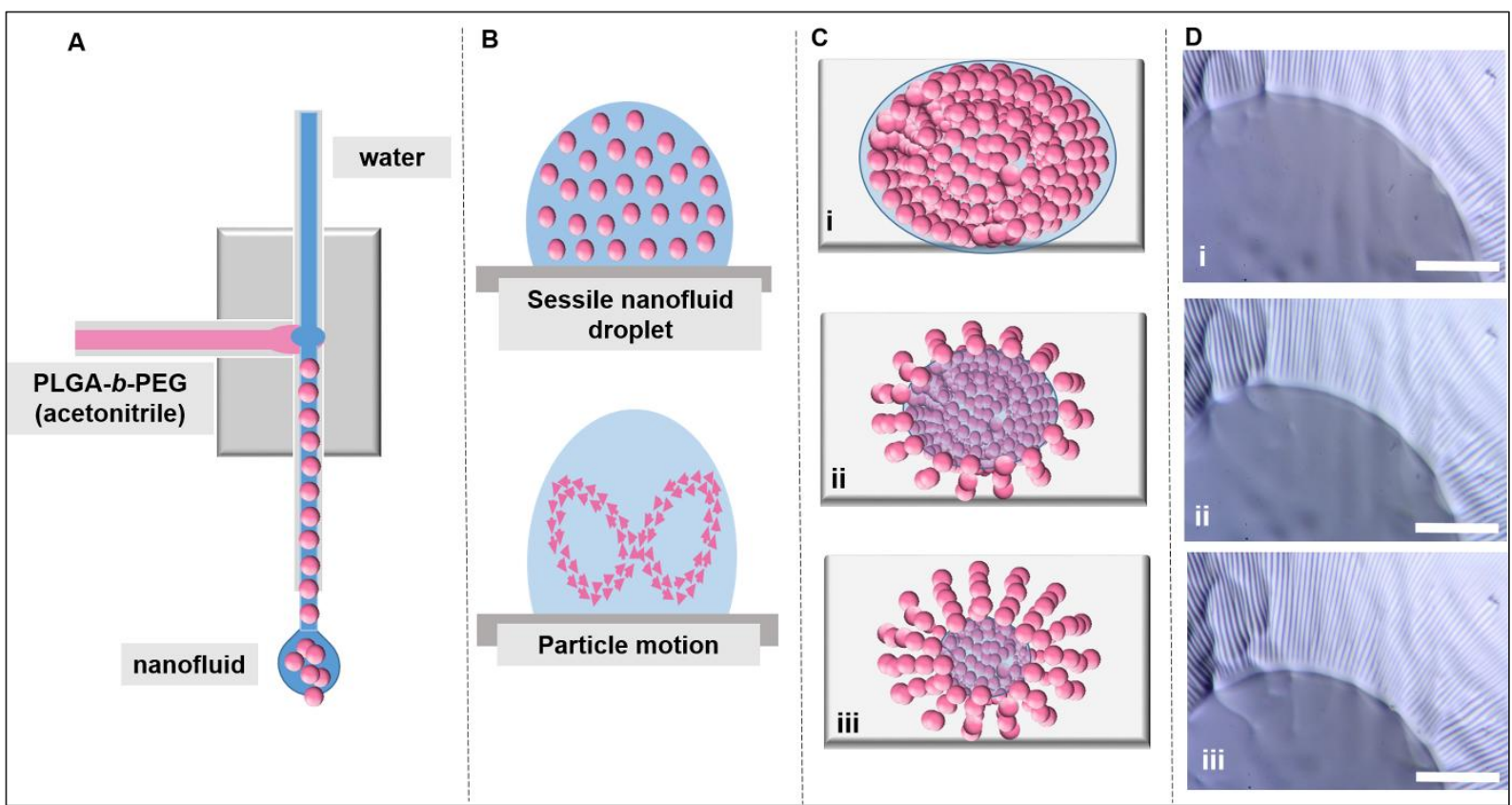

Figure 1. A) Schematic representation of PLGA- $b$-PEG nanoparticle based nanofluid production via hydrodynamic flow focusing, $B$ ) representative cross-sectional images of sessile nanofluid drop collection on glass slide and the particles motion inside the sessile droplet, C) representative image of i) sessile droplet, ii) drying and microstripe formation from orientated nanoparticles, iii) increased droplet drying and selfassembled pattern, D) optical microscope images of sessile droplet drying and microstripe formation, i-iii) shown step by step drying, scale bar $=200 \mu \mathrm{m}$. 
Characterization of Nanoparticles and Self Assembled Layers: Nanofluid (PLGAb-PEG nanoparticles dispersed in water-acetonitrile mixture) was characterized with small angle X-ray scattering (SAXS) and Dynamic Light Scattering (DLS) techniques (Malvern zetasizer ZS, UK). Nanoparticle size, zeta potential and polydispersity index were measured via DLS. Particle size was also determined on its native stage via SAXS. Nanoparticle crystallinity was checked by wide angle X-ray scattering (WAXS). SAXS/WAXS were performed using a GANESHA 300 XL SAXS/WAXS system equipped with a High Brilliance Micro focus source providing X-rays with a wavelength of $1.54 \AA$. A Pilatus $300 \mathrm{~K}$ solid-state photon-counting detector is located inside the vacuum chamber with full motorization. The change/movement between SAXS $(0.005 \leq q \leq 0.3)$ and WAXS $(2 \leq 2 \theta \leq 55)$ configuration can be achieved within 1 min to allow fast data collection $(q=4 \pi / \lambda(\sin \theta))$. Samples were contained in a custom designed glass cover transmission holder. Cost-friendly ACADEMY glass cover slips were used as background substrate.

Contact angle of nanofluid solution was measured by goniometer (Attension Theta, Biolin Scientific, Finland). Each sample contained an equal amount $(4 \mu \mathrm{L})$ of droplets collected on a glass slide. Contact angles were measured using an optical tensiometer (Attension Theta, Biolin Scientific, Finland) for 600 s to evaluate sessile drop drying motive and contact area evolution. Measurements were repeated three times for each sample. Morphological observations were performed with Optical Light Microscopy (Zeiss Axiotech, Germany with an attached Nikon Eclipse ME 600 camera, Japan), and Scanning Electron Microscopy (SEM, FEI Inspect S50, USA). Samples were coated with gold sputter prior to SEM analysis (Edwards, UK).

\section{Results and Discussion}


In the present study, nanofluids were produced by hydrodynamic flow focusing with serial concentrations of PLGA-b-PEG with $10 \%$ PEG content (PLGA-b-PEG 10 ) with acetonitrile as oil phase and PLGA-b-PEG nanoparticles were synthesized inside the microfluidic T-junction device. Then, PLGA-b-PEG nanoparticles were dried in a sessile droplet to generate self-assembled patterns. In the literature, most of the researchers focused on the self-assembling of nanoparticles with the aid of $\mathrm{pH}$, light, polarity, temperature, hydrophobicity, metallic interactions etc. ${ }^{24-26}$. Contrary to this, in this study we focused on the self-assembling patterns created by drying sessile droplets of polymeric nanoparticle suspensions. During nanoparticle generation, water and oil phases were fed into the $\mathrm{T}$-junction microfluidic device from different channels ( $x$ and $y$ directions) with different flow rates. Water phase was varied as 50 , 100 and $150 \mu \mathrm{L} / \mathrm{min}$ at constant flow rate of oil phase $(50 \mu \mathrm{L} / \mathrm{min})$. In the T-junction device, when water runs into the oil phase, PLGA-b-PEG precipitated directly in the water phase and nanoparticle formation occurred. It was found that PLGA-b-PEG concentration affected the nanoparticle properties, this was confirmed by both DLS and SAXS measurements (Table 1).

Table 1. The effect of PLGA- $b-P E_{10}$ (PEG 10 indicates PEG content in 10\%) concentration and the water/oil flow ratio in the microfluidic device on nanoparticle size (DLS and SAXS), PDI and zeta potential.

\begin{tabular}{cccccc}
$\begin{array}{c}\text { Polymer } \\
\%(\mathbf{w} / \mathbf{v})\end{array}$ & $\begin{array}{c}\text { Water/oil } \\
\text { Flow ratio }\end{array}$ & $\begin{array}{c}\text { Size } \\
\mathbf{D L S} \\
(\mathbf{n m})\end{array}$ & $\begin{array}{c}\text { Poly dispersity } \\
\text { index (PDI) }\end{array}$ & $\begin{array}{c}\text { Zeta } \\
\text { Potential } \\
(\mathbf{m V})\end{array}$ & $\begin{array}{c}\text { Size } \\
\text { SAXS } \\
(\mathbf{n m})\end{array}$ \\
\multirow{2}{*}{$\mathbf{2 . 5}$} & 1 & $140 \pm 1$ & $0.14 \pm 0.2$ & $-12.2 \pm 0.2$ & 141 \\
& 2 & $141 \pm 1$ & $0.13 \pm 0.1$ & $-8.1 \pm 0.4$ & 112 \\
& 3 & $140 \pm 1$ & $0.12 \pm 0.1$ & $-7.5 \pm 0.2$ & 128 \\
& 1 & $107 \pm 1$ & $0.06 \pm 0.01$ & $-8.5 \pm 0.4$ & 106 \\
$\mathbf{5}$ & 2 & $107 \pm 1$ & $0.05 \pm 0.01$ & $-8.5 \pm 0.5$ & 107 \\
& 3 & $108 \pm 1$ & $0.04 \pm 0.02$ & $-6.4 \pm 0.7$ & 109
\end{tabular}




$\begin{array}{lr}1 & 94 \pm 1 \\ 2 & 102 \pm 1 \\ 3 & 104 \pm 1\end{array}$

$0.15 \pm 0.2$

$-7.2 \pm 0.3$

From the DLS dataset collected under a constant flow ratio of $1: 1$, average nanoparticle size was determined as $140 \mathrm{~nm}$ for $2.5 \%$ PLGA-b-PEG 10 . It decreased to $107 \mathrm{~nm}$ for $5 \%$ PLGA- $b-\mathrm{PEG}_{10}$ and then further decreased to $94 \mathrm{~nm}$ for $10 \%$ PLGA- $b-P E G_{10}$. From the SAXS dataset of the same constant flow ratio, average nanoparticle size was determined as $141 \mathrm{~nm}$ for $2.5 \%$ PLGA- $b-P E G_{10}$, decreasing to

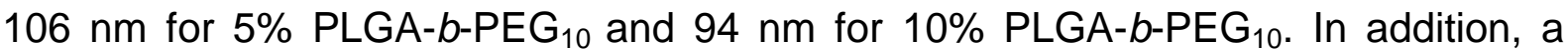
similar decreasing trend was observed at higher water/oil flow ratios which are also tabulated in Table 1. Average particle sizes were measured by DLS as $141 \mathrm{~nm}, 107$ $\mathrm{nm}$ and $102 \mathrm{~nm}$ at flow ratio 2 and $140 \mathrm{~nm}, 108 \mathrm{~nm}$ and $104 \mathrm{~nm}$ at flow ratio 3 for $2.5 \%, 5 \%$ and $10 \%$ PLGA- $b-\mathrm{PEG}_{10}$, respectively. Average particle sizes determined by SAXS were $112 \mathrm{~nm}, 107 \mathrm{~nm}$ and $94 \mathrm{~nm}$ at flow ratio 2 and $128 \mathrm{~nm}, 109 \mathrm{~nm}$ and $100 \mathrm{~nm}$ at flow rate ratio 3 for $2.5 \%, 5 \%$ and $10 \%$ PLGA- $b-\mathrm{PEG}_{10}$, respectively. Moreover, increasing polymer concentration caused an increase in zeta potential values. Zeta potential value was $-12.2 \mathrm{mV}$ for $2.5 \%$ PLGA- $b-P E G_{10},-8.5 \mathrm{mV}$ for $5 \%$ PLGA- $b-$ PEG $_{10}$ and $-7.2 \mathrm{mV}$ for $10 \%$ PLGA- $b-\mathrm{PEG}_{10}$ at a water/oil flow ratio of 1 (50:50 $\mu \mathrm{L} / \mathrm{min})$. It was concluded that increasing polymer concentration and water/oil ratio decreased size and increased zeta potential of nanoparticles (Figure 1A-B). Nanoparticle sizes measured by DLS in solution are larger than those measured by SAXS in the solid state (Figure 1C). This is likely to be caused by the continuous growth of nanoparticles in the liquid state caused by prolonged interaction time between PEG hydrophilic chains and water. Cheng et al. $^{27}$ also concluded that PLGA- $b-\mathrm{PEG}_{10}$ nanoparticle size varies at different polymer concentrations in organic 
solvent but at a fixed PLGA- $b-P \mathrm{G}_{10}$ concentration and water/oil phase, difference in

particle sizes are very slight.

It is also observed that obtained PLGA-b-PEG 10 nanoparticles are highly monodispersed, and the polydispersity index (PDI) ranges between $0.17-0.04$. In the literature, PLGA-b-PEG 10 nanoparticles PDI is generally around $0.20^{28-29}$. In our case, especially $5 \%$ PLGA-b-PEG 10 , highly monodisperse nanoparticles with $0.06,0.05$ and $0.04 \mathrm{PDI}$ values for 1,2 and 3 water/oil flow rates, respectively, were generated which is a very remarkable new result for the literature. It was concluded that, water/oil flow ratio did not exhibit a significant difference on nanoparticle size, PDI and zeta potential, polymer concentration of PLGA- $b-P_{E} G_{10}$ was the most affective factor.
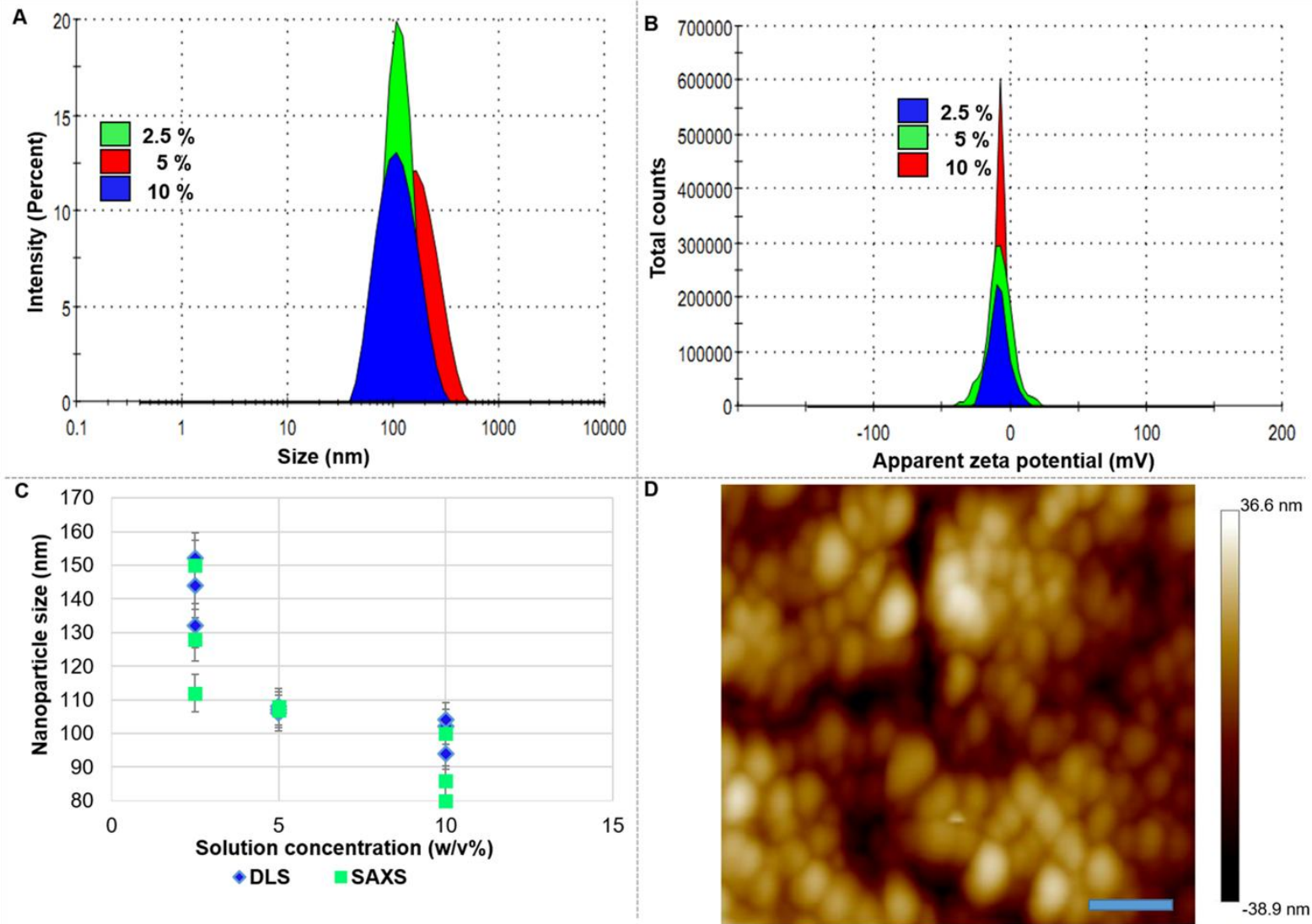

Figure 2. PLGA-b-PEG 10 nanoparticles $A$ ) average particle size $(n m)$, B) zeta potential $(\mathrm{mV})$ distribution graphs of $2.5 \%, 5 \%$ and $10 \%$ PLGA- $b$-PEG solutions (50:50 oil/water flow ratio), C) comparative size distribution graph of $2.5 \%, 5 \%$ and 
$10 \%$ PLGA- $b$-PEG solutions, D) AFM micrograph of PLGA- $b$-PEG nanoparticles, scale bar $=200 \mathrm{~nm}$ (50:50 oil/water flow ratio, $5 \%(\mathrm{w} / \mathrm{v})$ concentration).

Comparative size (Figure 2A) and zeta potential (Figure 2B) graphs represent the concentration gradient for $5 \%$ PLGA- $b-\mathrm{PEG}_{10}$ at flow ratio 1 . In addition, PLGA- $b$ $P E G_{10}$ nanoparticles size and morphology were also investigated by AFM. Figure 2D shows the AFM micrograph of highly monodisperse nanoparticles of $5 \%$ PLGA- $b$ $\mathrm{PEG}_{10}$ at flow ratio 1. AFM micrograph proved that the synthesized nanoparticles have spherical morphology and their size is around $100 \mathrm{~nm}$ diameter.



Figure 3. SAXS/WAXS measurements of PLGA- $b-P_{E G_{10}}$ nanoparticles formed in $5 \%$ $(w / v)$ solution at flow rate ratio 1 (oil:water) A) Log-log plot of SAXS pattern, B) Lin-lin plot of WAXS pattern shown in 2-theta configuration, C) full fit curve matched with data after subtracting background, D) 2D scattering pattern collected in SAXS, E) 2D scattering pattern collected in WAXS configuration.

It is known that results from SEM and DLS involve different kinds of post-synthesis operations, this may have a huge impact on the particle size. For this reason, we employed SAXS/WAXS technique with a specially-designed thin-film cell holder to examine the particles in their native state on the glass cover, following pattern 
formation and drying. No grinding, post-preparation or any disturbance was introduced to the sample prior to the SAXS measurements. We used a custom-made sample holder fitted with ACADEMY background cover slips to obtain structural information of the thin film nanoparticles in their native state. The difference between red curve (scattering from the sample) and blue curve (scattering from the blank glass cover, which is subtracted as the background) showed that that the noise-tosignal ratio is very low and therefore we could collect strong SAXS signals to reveal true features of the nanoparticles (Figure $3 \mathrm{~A}$ ). It proved that this cost-effective cover slides are very suitable substrates for nanoparticle thin films, also making them good alternatives to scatterless MICA/Kapton films which were widely used as window/background materials in SAXS measurements. Here, we used 'SAXSGUI' to do 'Full Fit' from the background subtracted data to obtain particle size information (Figure 3C). A polydispersed sphere model was used to fit the curve, and a Gaussian distribution was applied as it is known that the nanoparticles are spherical with some degree of polydispersity (5\% polydispersity from DLS measurement). The particle size determined by SAXS $(106 \mathrm{~nm})$ is smaller than those obtained from the DLS $(107 \mathrm{~nm})$ analysis. This very small difference is expected because DLS gives hydrodynamic radius which is known to be bigger than the so called 'true radius' measured from SAXS or microscopy. This indicated that in the process of the selfassembling and specific pattern formation, there is subtle change (or even no change) of these nanoparticle sizes from suspension state to solid state. The WAXS curve collected (Figure 3D) showed a typical amorphous scattering pattern with a very broad (amorphous) hump.

It is clear that PLGA-b-PEG nanoparticles remained amorphous during selfassembling and pattern formation processed. The observed high stability of this 
amorphous property is often key and preferred for optimising drug delivery, as unwanted crystallization or low bioavailability/dissolution rate can have a huge impact on drug safety, drug patent protection and other crucial issues in pharmaceutical industries. There have been several studies with forming polymeric, especially PLGA$b$-PEG nanoparticles ${ }^{30}$, nevertheless this is the first report studying sessile nanodroplet drying with self-assembled pattern structures of PLGA- $b$-PEG. SAXS/WAXS analyses showed that the particles retained their size and shape during the drying of sessile nanofluid droplets. Additionally, we investigated the sessile nanofluid droplets dried pattern structures. An additional data set with fixed flow ratio (1) and varying polymer concentrations (2.5, 5, $10 \%)$ was also studied to investigate the effect of PEG block content on the sessile droplet pattern, thus taking in to account PEG content, nanoparticle size, PDI and zeta potential. PLGA-b-PEG with $15 \%$ PEG content (PLGA-b-PEG ${ }_{15}$ ) exhibited higher hydrodynamic nanoparticle size with lower zeta potential values as a result of increasing PEG chain length. PDI values were also increased compare to values obtained from PLGA-b-PEG (Table 2).

Table 2. The effect of PEG content (15 in compare to 10 in Table 1) and polymer concentration on nanoparticles size, PDI and zeta potential.

$\begin{array}{ccccc}\begin{array}{c}\text { Polymer \% } \\ (\mathbf{w} / \mathbf{v})\end{array} & \begin{array}{c}\text { Water/oil } \\ \text { Flow ratio }\end{array} & \begin{array}{c}\text { Size } \\ \text { DLS } \\ (\mathbf{n m})\end{array} & \begin{array}{c}\text { Poly dispersity } \\ \text { index (PDI) }\end{array} & \begin{array}{c}\text { Zeta Potential } \\ (\mathbf{m V})\end{array} \\ \mathbf{2 . 5} & 1 & 175 \pm 1 & 0.16 \pm 0.1 & -1.9 \pm 0.1 \\ \mathbf{5} & 1 & 203 \pm 1 & 0.15 \pm 0.2 & -1.5 \pm 0.1 \\ \mathbf{1 0} & 1 & 266 \pm 2 & 0.24 \pm 0.1 & -0.8 \pm 0.2\end{array}$


An evaporating sessile droplet is characterized by its height, radius and contact angle values. Contact angle values of PLGA-b-PEG nanofluids were determined by sessile drop technique on a glass slide. Because of using a glass slide as the collector during the whole study it is important to determine and compare solution characteristics on the same surface. The evaporation mode of sessile droplet is classified by the change in one or some of these attributes during the drying process $^{31}$. As can be seen in Figure 3 the contact line is pinned in all PLGA-b-PEG 10 content keeping the radius of the contact area constant while a decrease in height is observed: in constant contact radius (CCR) mode, a linear contact angle decreases with linear volume loss. We believe that this two-step decreases are a result of a binary liquid system, occurring with the fast evaporation of volatile acetonitrile followed by water. Nevertheless, the evaporation of PLGA-b-PEG ${ }_{15}$ sessile droplets starts with the CCR mode, however it turns into constant contact angle (CCA) mode at 5 and 10 percentages as the radius decreases while the contact angle changes after 120s (Figure 4). 


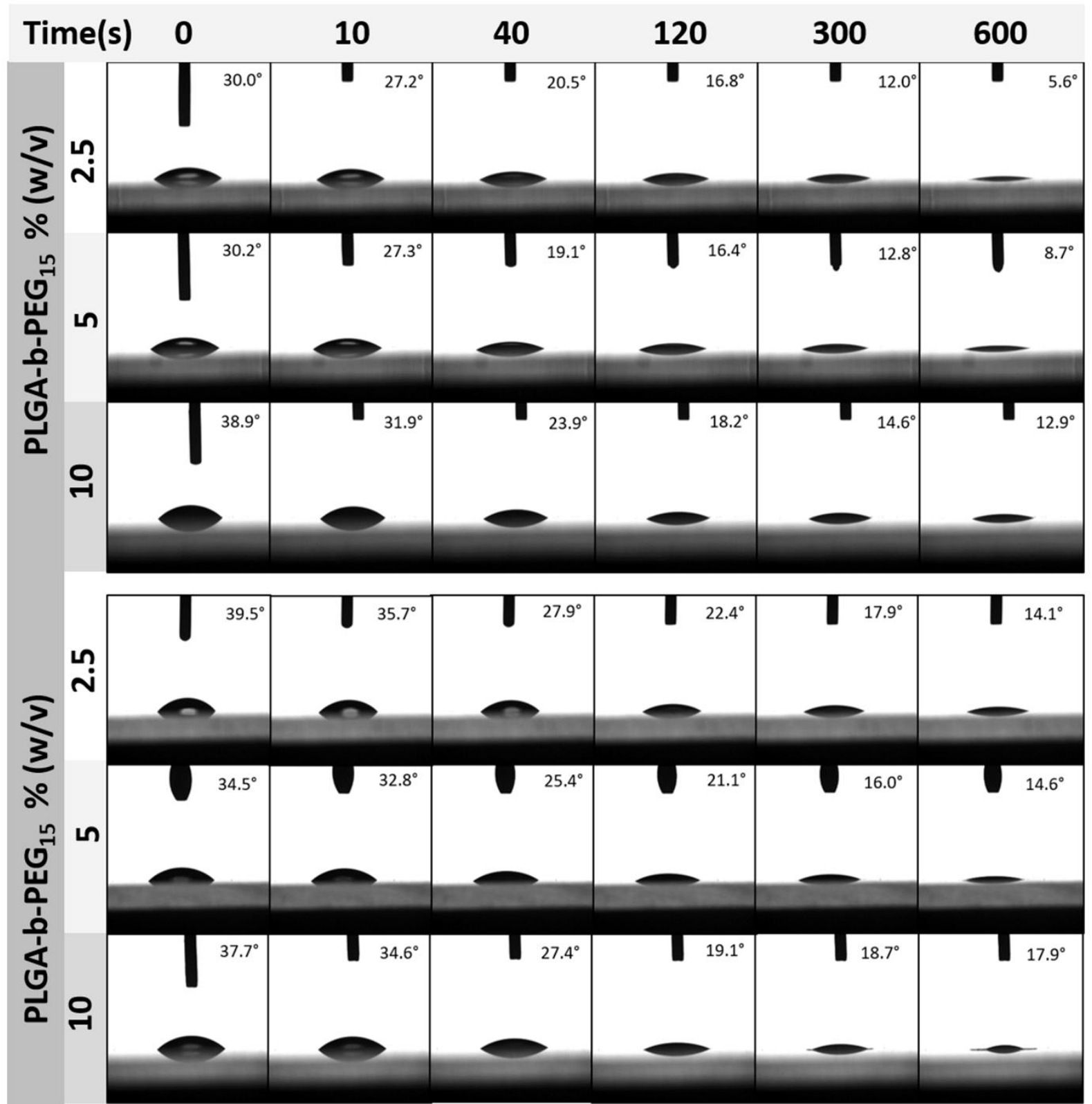

Figure 4. Contact angle values of PLGA- $b-P E G_{10}$ and PLGA- $b-P E G_{15}$ nanofluids of $2.5,5$ and $10 \%(\mathrm{w} / \mathrm{v})$ concentration at $\mathrm{t}=0,10,40,120,300,600 \mathrm{~s}$ of sessile droplet drying time periods.

The contact angle of PLGA- $b$-PEG 10 droplet is descending (Figure $5 \mathrm{~A}$ ) whereas, it remains the same in PLGA-b-PEG ${ }_{15}$ (Figure $5 B$ ). CCA phenomenon was previously reported by Erbil et al. ${ }^{32}$ with a diminishing drop height and area of contact. Small variations in contact angle values can be observed as McHale et al. ${ }^{33}$ reported, which is also seen in our study. However, they indicated that contact angle is a slow variable compared to area of contact. CCA angle mode is typically seen in smooth 
hydrophobic surfaces and the reason of this mode change can be caused by the high



Figure 5. Contact angle versus time variation for 2.5, 5 and $10 \%(w / v)$ A) PLGA- $b$ $P G_{10}$ and $\left.B\right)$ PLGA- $b-P E G_{15}$ sessile droplet nanofluids.

CCA type evaporation mode indicates that at higher concentrations of PLGA-b$\mathrm{PEG}_{15}$ nanoparticles tend to deposit on the hydrophilic glass slide rather than move inside the droplet by internal flow, which can be interpreted by increasing hydrodynamic radius and neutralized zeta potentials compared to the same concentrations of PLGA-b-PEG 10 . Decreasing repelling forces and increased size of nanoparticles caused aggregation during the collision of the particles inside the droplet creating a hydrophobic layer. Vafaei et al. ${ }^{34}$ studied the effect of nanofluid concentration on surface wettability and stated that the contact angle of nanofluids increase with increasing concentration. Similarly, in our case contact angle of PLGA$b-P E G_{10}$ which have $C C R$ type contact angle increases with increasing concentration. 
The drying of nanofluid systems with CCR usually results with coffee stain patterns after the suppression of Marangoni effect ${ }^{35}$. However, uniform deposition of nanoparticles, especially at high densities, was also occasionally reported ${ }^{36-37}$. The denser population of nanoparticles with high viscosity suppresses the thermally induced Marangoni effect, resulting in a uniform thicker pattern in the contact area. Moreover, the contribution of faster evaporation on Marangoni effect is also stated in the literature; Bigioni et al. $^{38}$ reported an exceptional long range ordered nanoparticles by enhanced evaporation rate. Marangoni flow recirculates the nanoparticles inside the droplet, preventing them to deposit at the pinned edge. Concordantly, accumulation of nanoparticles at the air-liquid interface rather than drop edge is also possible by increasing the evaporation as reported by Li et al. ${ }^{1}$ who took into account that, the above mentioned examples include metallic and inorganic based nanofluids with single liquid systems. In our study, binary liquid system (wateracetonitrile) evaporates sequentially and with the aid of sequential drying motion the nanoparticles are evenly distributed inside the droplet caused by the Marangoni effect. 


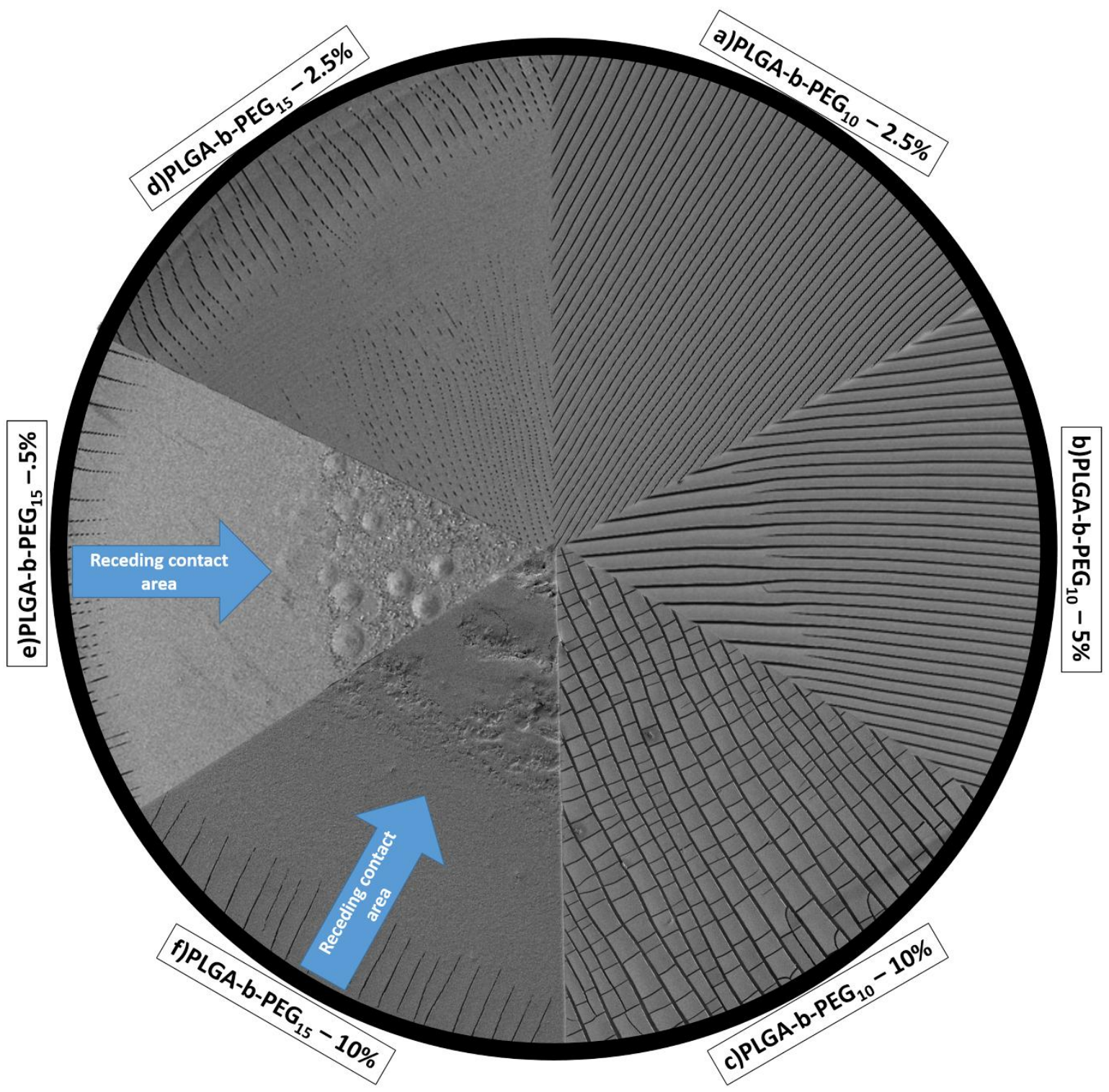

Figure 6. Scanning electron microscope images of self-assembled micro-stripe patterns from PLGA- $b$-PEG 10 and PLGA- $b$-PEG ${ }_{15}$ sessile nanofluid droplets produced at oil/water flow ratio 1 with different polymer concentrations. a) $2.5 \%$ b) $5 \%$ c) $10 \%$ (w/v) PLGA-b-PEG 10 and d) $2.5 \%$ e) 5\% f) $10 \%$ (w/v) PLGA-b-PEG ${ }_{15}$.

The sessile droplets dried at ambient conditions and different patterns were discovered for different PLGA- $b-\mathrm{PEG}_{10}$ and PLGA- $b-\mathrm{PEG}_{15}$ polymer concentrations. The obtained patterns were investigated by Scanning Electron Microscope (SEM) are summarised in Figure 6A-F. Evaporation of sessile nanofluid droplets is driven by two main flow regimes which are Capillary flow and Marangoni flow ${ }^{35,}$ 39. Here, the nanofluid droplet which was prepared from 2.5, 5 and 10\% (w/v) PLGA-b-PEG 10 
nanofluids created self-assembled micro-stripe patterns by highly ordered radial crack formation (Figure 5A-C). In Figure 5D-F, 2.5, 5 and 10\% (w/v) PLGA-b-PEG 15 nanofluid sessile droplet drying patterns were investigated and it is seen that the cracks at the drop edge started to form in a similar way but were interrupted or completely disappeared later on. The latter case was seen on 5 and $10 \%$ which has also CCA type drying pattern indicating that crack formation did not occur on deposited layer of nanoparticles with larger size and lower zeta potential.

The reason for crack formation at the droplet edge is a result of two competing forces, substrate adhesion and deposit shrinkage through evaporation. As the droplet shrinks while creating a local stress from the edge, the adhesion force between the droplet and substrate compresses the structure. Crack formation starts at the edge as a result of increased tensile strength. In our study, receding of contact area is followed by crack formation in the final stage of evaporation, which is remarkable and dominant particularly in PLGA-b-PEG 10 samples, towards the central regions. The direction of the cracks is similar at all polymer concentrations, however the width between the adjoining cracks (micro-stripes) increases with concentration.

The uniform micro-stripe formation was monitored by optical microscope in a time dependent manner in Figure 6 for $5 \%(\mathrm{w} / \mathrm{v})$ PLGA-b-PEG 10 samples. Stripes propagate continuously by $\sim 100 \mathrm{~nm}$ nanoparticles at $2 \mathrm{~s}$ intervals from Figure 7-A to 7-H. Stripes have uniform morphology in terms of both linearity and diameter with the assistance of Marangoni recirculation inside the sessile nanofluid droplet. Crack formation is observed by light microscopy as the wet front recedes and it causes an instability in the liquid zone followed by crack micro-stripe patterning. 
$\bar{E}$

$\bar{F}$

$\bar{G}$

Figure 7. Optical microscope images of $5 \%(\mathrm{w} / \mathrm{v})$ PLGA-b-PEG 10 sessile nanofluid droplets drying as a function of time, scale bar $=50 \mu \mathrm{m}(\mathrm{A}-\mathrm{H}$, time intervals $=2 \mathrm{~s}$, oil/water flow ratio 1 ).

Gorr et al..$^{40}$ stated that the width and the direction of the cracks depends on the drop shape evolution. In our experiments, we observed that drying sessile drops obeying the CCR type has well-ordered crack formation and propagation through the radial direction. The crack formation was also observed in PLGA-b-PEG ${ }_{15}$ sample groups, however the propagation in the radial direction continues only in $2.5 \%$ concentration with an interruption. Crack formation at the edges was also noticed at higher densities of PLGA-b-PEG ${ }_{15}$, though the crack propagation disappeared after the ring area indicating the tensile stress does not reach the local tensile strength. Additionally, multiple distinct bumps and irregular intrusions were also seen in 5 and $10 \%(\mathrm{w} / \mathrm{v})$ concentrations of PLGA-b-PEG ${ }_{15}$, respectively. Amongst the six patterns shown in Figure 6, nanoparticles created the most uniform, highly ordered and continuous micro-stripe patterns along the evaporation direction. In Figure $6 \mathrm{C}$ the tensile strength during evaporation is so high that orthoradial cracks were also observed in the obtained pattern. 
The uniform crack formations and the nanoparticles embedded in micro-stripes are shown in Figure 8A-F. Also, embedded nanoparticles were shown in Figures 8D-F where the nanoparticle size is confirmed by SEM, AFM, SAXS/WAX and DLS measurements as $\sim 100 \mathrm{~nm}$. Radial crack width increases with the nanoparticle concentration. This tendency can be explained by $2.5 \%(\mathrm{w} / \mathrm{v})$ PLGA-b-PEG 10 (Figure $8 \mathrm{~A}$ ) has the thinner uniform linear patterns than $5 \%(\mathrm{w} / \mathrm{v})$ PLGA-b-PEG 10 (Figure 8B) and $10 \%(w / v)$ PLGA-b-PEG 10 (Figure $8 C$ ) has linear patterns with orthoradial linear cracks since it has the highest polymer concentration (10\%) and the highest concentration of nanoparticles in an equal amount of sessile droplet amongst the samples. The width of micro-stripes was measured as $8.5 \pm 0.2,14.2 \pm 0.3$ and $14.8 \pm 0.3 \mu \mathrm{m}$ for $2.5,5$ and $10 \%$ (w/v) PLGA-b-PEG 10 , respectively (Figure $8 A-C$ ).

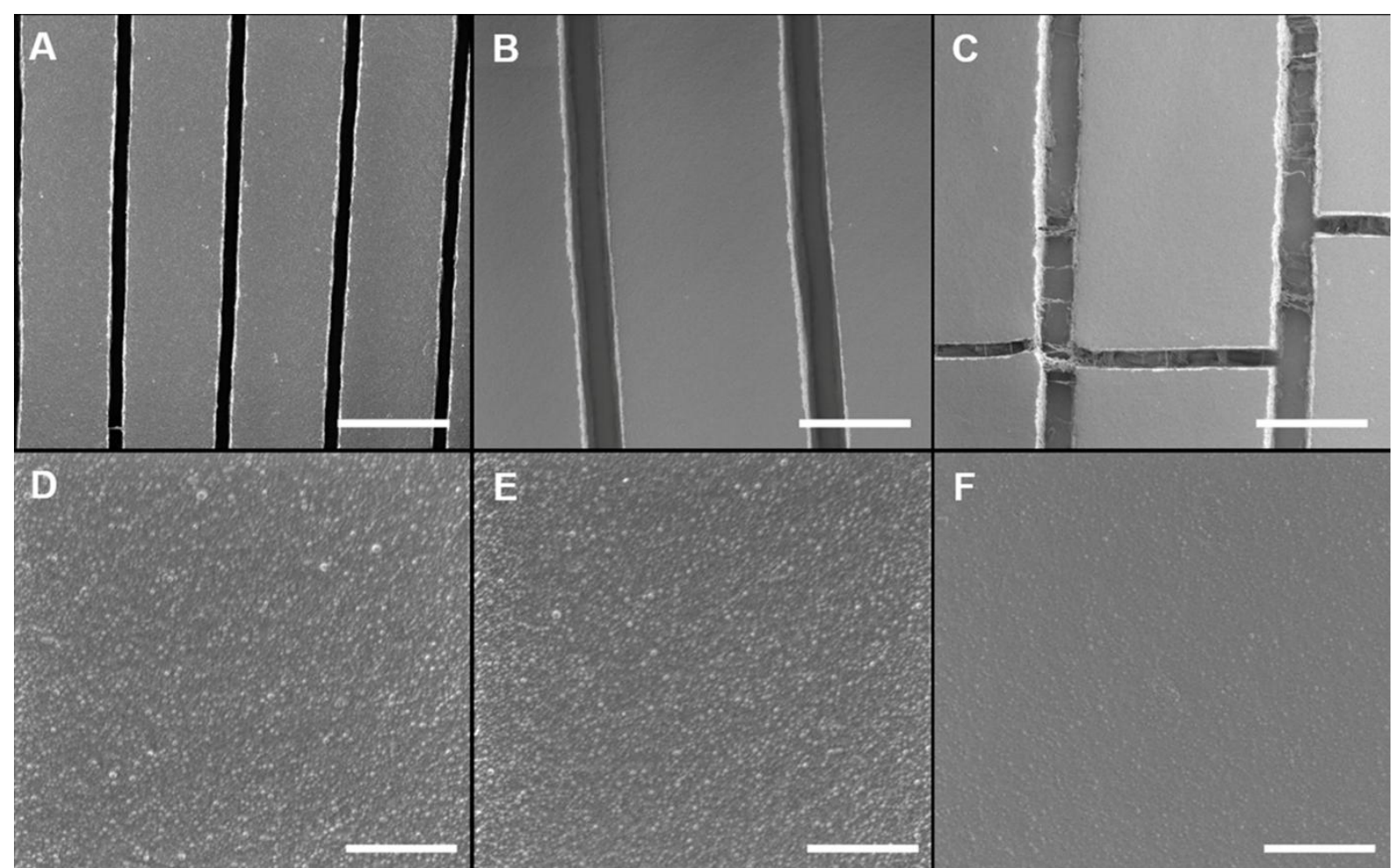

Figure 8. Scanning electron microscope images of self-assembled patterns from PLGA- $b-\mathrm{PEG}_{10}$ sessile nanofluid droplets produced at oil/water flow ratio 1 with different polymer concentrations A) 2.5, B) 5 , C) $10 \%$ (w/v) PLGA-b-PEG 10 (scale bar $=10 \mu \mathrm{m}$ ) and high magnification micrographs of embedded nanoparticles of $D$ ) 2.5 , E) 5, F) $10 \%$ (w/v) PLGA-b-PEG 10 (scale bar= $2 \mu \mathrm{m}$ ). 
Deegan et al. ${ }^{8}$ reported that coffee-ring deposition is forced by Capillary flow which starts from a pinned contact line. Moreover, $\mathrm{Hu}$ and Larson $^{9}$ explained that the Marangoni effect should be supressed to obtain coffee-ring deposits, because the Marangoni effect forces the creation of self-assembled highly ordered patterns ${ }^{41-42}$. Here, sessile drops of nanofluid solutions dried sequentially. Vapour pressure of acetonitrile is higher than water, hence acetonitrile evaporates first. Acetonitrile concentration becomes higher at the edge of sessile droplet and reduces the surface tension of the droplet. Marangoni flow was induced by the change in surface tension and recirculation occurred inside the sessile droplet. The Stokes equation (Equation 1) gives the relationship between the gravitational force, settling velocity, density, viscosity and particle size ${ }^{43}$.

$$
V S=\frac{2}{9} \frac{\left(\rho_{p}-\rho_{f}\right)}{\mu} g R^{2}
$$

where Vs is the settling velocity, $\rho_{p}$ is particle density, $\rho_{f}$ is the nanofluid density, $\mu$ is nanofluid dynamic viscosity, $g$ is gravity and $R$ is the radius of the nanoparticles.

Marangoni effect was suppressed partially for 2.5 and totally for $5,10 \%(w / v)$ PLGA$b$-PEG ${ }_{15}$ samples and uniform deposition was hindered. Besides, all concentrations of PLGA- $b-\mathrm{PEG}_{10}$ resulted in ordered micro-stripe patterning due to the Marangoni flow with the optimal particle size for self-assembly patterns. In the present study, stripe formation threshold was found to be $\sim 175 \mathrm{~nm}$ for PLGA- $b$-PEG nanoparticles whereas uniform and continuous formation occurred at $\sim 100 \mathrm{~nm}$. Moreover, effect of zeta potential on uniform micro-stripe formation is dominant. Cai et al. ${ }^{44}$ also reported that $100 \mathrm{~nm}$ polystyrene nanoparticles were assembled into stripe like patterns with Marangoni flow. This particle size threshold can be explained using Equation 1, 
because nanoparticle circulation velocity is proportional to the square of particle radius. Moreover, velocity and flow type was affected not only by particle size but also by density of nanoparticles and nanofluids. For instance, 5 and 10\% (w/v) PLGA-b-PEG have the same particle size and they both deposited as micro-stripe patterning. Besides, $5 \%$ samples have highly ordered micro-stripe patterns due to the lower particle and nanofluid density gradient (Equation 1). It was also concluded that highly monodisperse (relatively low PDI) and highly charged (zeta potential) particles deposited as thinner stripes (Figure 6A-B and Figure 8A-C).

\section{Conclusions}

In this study, highly monodisperse PLGA-b-PEG nanoparticles were obtained by surfactant free hydrodynamic flow focusing in a T-junction microfluidic device. Synthesized nanoparticles were collected as nanofluid solutions and the nanofluids were dried as sessile droplets. Due to the internal flow type of the sessile droplets different deposition patterns were obtained. Internal flow type and the related deposition patterns were affected by the polymer concentration, nanoparticles size, charge and polydispersity index. PLGA-b-PEG nanoparticles which have extremely low PDI and $100 \mathrm{~nm}$ particle size were deposited as highly uniform self-assembled parallel stripes. Thus, T-junction microfluidic devices are ideal systems for the surfactant free, highly monodisperse nanoparticle fabrication and these nanoparticles exhibit highly ordered and self-assembled stripe patterns under optimized conditions.

\section{Acknowledgements}

This study was financially supported by Hacettepe University, Scientific Research Projects Coordination Unit (Grant Numbers: TDK-2017-14725 and FBI-2017-14296). 


\section{References}

1. Li, C. H.; Peterson, G., Experimental investigation of temperature and volume fraction variations on the effective thermal conductivity of nanoparticle suspensions (nanofluids). Journal of Applied Physics 2006, 99 (8), 084314.

2. Xie, H.; Lee, H.; Youn, W.; Choi, M., Nanofluids containing multiwalled carbon nanotubes and their enhanced thermal conductivities. Journal of Applied Physics 2003, 94 (8), 4967-4971.

3. Fang, X.; Ding, Q.; Fan, L.-W.; Lu, H.; Yu, Z.-T., Effects of inclusion size on thermal conductivity and rheological behavior of ethylene glycol-based suspensions containing silver nanowires with various specific surface areas. International Journal of Heat and Mass Transfer 2015, 81, 554-562.

4. Lee, K. J.; Yoon, S. H.; Jang, J., Carbon nanofibers: a novel nanofiller for nanofluid applications. Small 2007, 3 (7), 1209-1213.

5. Shen, X.; Ho, C.-M.; Wong, T.-S., Minimal size of coffee ring structure. The Journal of Physical Chemistry B 2010, 114 (16), 5269-5274.

6. Ryu, S.-a.; Kim, J. Y.; Kim, S. Y.; Weon, B. M., Drying-mediated patterns in colloid-polymer suspensions. Scientific Reports 2017, 7 (1), 1079.

7. Han, W.; Lin, Z., Learning from "Coffee Rings": Ordered Structures Enabled by Controlled Evaporative Self-Assembly. Angewandte Chemie International Edition 2012, 51 (7), 1534-1546.

8. Deegan, R. D.; Bakajin, O.; Dupont, T. F.; Huber, G.; Nagel, S. R.; Witten, T. A., Capillary flow as the cause of ring stains from dried liquid drops. Nature 1997, 389 (6653), 827.

9. Hu, H.; Larson, R. G., Marangoni effect reverses coffee-ring depositions. The Journal of Physical Chemistry B 2006, 110 (14), 7090-7094.

10. Sangani, A. S.; Lu, C.; Su, K.; Schwarz, J. A., Capillary force on particles near a drop edge resting on a substrate and a criterion for contact line pinning. Physical Review E 2009, 80 (1), 011603.

11. Harris, D. J.; Conrad, J. C.; Lewis, J. A., Evaporative lithographic patterning of binary colloidal films. Philosophical Transactions of the Royal Society of London A: Mathematical, Physical and Engineering Sciences 2009, 367 (1909), 5157-5165.

12. Layani, M.; Gruchko, M.; Milo, O.; Balberg, I.; Azulay, D.; Magdassi, S., Transparent conductive coatings by printing coffee ring arrays obtained at room temperature. ACS nano 2009, 3 (11), 3537-3542.

13. Vohra, V.; Bolognesi, A.; Calzaferri, G.; Botta, C., Self-assembled nanofibers of fluorescent zeolite L crystals and conjugated polymer. Langmuir 2010, 26 (3), 1590-1593.

14. Smalyukh, I. I.; Zribi, O. V.; Butler, J. C.; Lavrentovich, O. D.; Wong, G. C., Structure and dynamics of liquid crystalline pattern formation in drying droplets of DNA. Physical Review Letters 2006, 96 (17), 177801.

15. Whitesides, G. M., The origins and the future of microfluidics. Nature 2006, 442 (7101), 368-

73.

16. Valencia, P. M.; Farokhzad, O. C.; Karnik, R.; Langer, R., Microfluidic technologies for accelerating the clinical translation of nanoparticles. Nature Nanotechnology 2012, 7 (10), 623.

17. Ali, H. S.; York, P.; Ali, A. M.; Blagden, N., Hydrocortisone nanosuspensions for ophthalmic delivery: a comparative study between microfluidic nanoprecipitation and wet milling. Journal of Controlled Release 2011, 149 (2), 175-181.

18. Liu, D.; Cito, S.; Zhang, Y.; Wang, C. F.; Sikanen, T. M.; Santos, H. A., A versatile and robust microfluidic platform toward high throughput synthesis of homogeneous nanoparticles with tunable properties. Advanced Materials 2015, 27 (14), 2298-2304.

19. Valencia, P. M.; Basto, P. A.; Zhang, L.; Rhee, M.; Langer, R.; Farokhzad, O. C.; Karnik, R., Single-step assembly of homogenous lipid- polymeric and lipid- quantum dot nanoparticles enabled by microfluidic rapid mixing. ACS nano 2010, 4 (3), 1671-1679.

20. Valencia, P. M.; Pridgen, E. M.; Rhee, M.; Langer, R.; Farokhzad, O. C.; Karnik, R., Microfluidic platform for combinatorial synthesis and optimization of targeted nanoparticles for cancer therapy. ACS nano 2013, 7 (12), 10671-10680. 
21. Elsayed, M.; Kothandaraman, A.; Edirisinghe, M.; Huang, J., Porous Polymeric Films from Microbubbles Generated Using a T-Junction Microfluidic Device. Langmuir 2016, 32 (50), 1337713385.

22. Gultekinoglu, M.; Jiang, X.; Bayram, C.; Ulubayram, K.; Edirisinghe, M., Honeycomb-like PLGAb-PEG Structure Creation with T-junction Micro Droplets. Langmuir 2018, 34 (27), 7989-7997.

23. Bayram, C.; Jiang, X.; Gultekinoglu, M.; Ozturk, S.; Ulubayram, K.; Edirisinghe, M. J. M. M.; Engineering, Biofabrication of Gelatin Tissue Scaffolds with Uniform Pore Size via Microbubble Assembly. Macromolecular Materials and Engineering 2019, 1900394.

24. Grzelczak, M.; Vermant, J.; Furst, E. M.; Liz-Marzán, L. M., Directed self-assembly of nanoparticles. ACS nano 2010, 4 (7), 3591-3605.

25. Chen, Y.; Wang, Z.; He, Y.; Yoon, Y. J.; Jung, J.; Zhang, G.; Lin, Z., Light-enabled reversible selfassembly and tunable optical properties of stable hairy nanoparticles. Proceedings of the National Academy of Sciences 2018, 201714748.

26. Sánchez-Iglesias, A.; Grzelczak, M.; Altantzis, T.; Goris, B.; Perez-Juste, J.; Bals, S.; Van Tendeloo, G.; Donaldson Jr, S. H.; Chmelka, B. F.; Israelachvili, J. N., Hydrophobic interactions modulate self-assembly of nanoparticles. ACS nano 2012, 6 (12), 11059-11065.

27. Cheng, J.; Teply, B. A.; Sherifi, I.; Sung, J.; Luther, G.; Gu, F. X.; Levy-Nissenbaum, E.; RadovicMoreno, A. F.; Langer, R.; Farokhzad, O. C., Formulation of functionalized PLGA-PEG nanoparticles for in vivo targeted drug delivery. Biomaterials 2007, 28 (5), 869-876.

28. Baysal, I.; Ucar, G.; Gultekinoglu, M.; Ulubayram, K.; Yabanoglu-Ciftci, S., Donepezil loaded PLGA-b-PEG nanoparticles: their ability to induce destabilization of amyloid fibrils and to cross blood brain barrier in vitro. Journal of Neural Transmission 2017, 124 (1), 33-45.

29. Sari, E.; Tunc-Sarisozen, Y.; Mutlu, H.; Shahbazi, R.; Ucar, G.; Ulubayram, K., ICAM-1 targeted catalase encapsulated PLGA-b-PEG nanoparticles against vascular oxidative stress. Journal of Microencapsulation 2015, 32 (7), 687-698.

30. Şimşek, S.; Eroğlu, H.; Kurum, B.; Ulubayram, K., Brain targeting of Atorvastatin loaded amphiphilic PLGA-b-PEG nanoparticles. Journal of Microencapsulation 2013, 30 (1), 10-20.

31. Zhang, X.; Wang, J.; Bao, L.; Dietrich, E.; van der Veen, R. C.; Peng, S.; Friend, J.; Zandvliet, H. J.; Yeo, L.; Lohse, D., Mixed mode of dissolving immersed nanodroplets at a solid-water interface. Soft Matter 2015, 11 (10), 1889-1900.

32. Erbil, H. Y.; McHale, G.; Newton, M., Drop evaporation on solid surfaces: constant contact angle mode. Langmuir 2002, 18 (7), 2636-2641.

33. McHale, G.; Rowan, S. M.; Newton, M.; Banerjee, M. K., Evaporation and the wetting of a low-energy solid surface. The Journal of Physical Chemistry B 1998, 102 (11), 1964-1967.

34. Vafaei, S.; Borca-Tasciuc, T.; Podowski, M.; Purkayastha, A.; Ramanath, G.; Ajayan, P., Effect of nanoparticles on sessile droplet contact angle. Nanotechnology 2006, 17 (10), 2523.

35. Parsa, M.; Harmand, S.; Sefiane, K., Mechanisms of pattern formation from dried sessile drops. Advances in Colloid and Interface Science 2018.

36. Sefiane, K., On the role of structural disjoining pressure and contact line pinning in critical heat flux enhancement during boiling of nanofluids. Applied Physics Letters 2006, 89 (4), 044106.

37. Giorgiutti-Dauphiné, F.; Pauchard, L., Drying drops. The European Physical Journal E 2018, 41 (3), 32 .

38. Bigioni, T. P.; Lin, X.-M.; Nguyen, T. T.; Corwin, E. I.; Witten, T. A.; Jaeger, H. M., Kinetically driven self assembly of highly ordered nanoparticle monolayers. Nature Materials 2006, 5 (4), 265270.

39. Karpitschka, S.; Liebig, F.; Riegler, H., Marangoni Contraction of Evaporating Sessile Droplets of Binary Mixtures. Langmuir 2017, 33 (19), 4682-4687.

40. Gorr, H. M.; Zueger, J. M.; McAdams, D. R.; Barnard, J. A., Salt-induced pattern formation in evaporating droplets of lysozyme solutions. Colloids and Surfaces B: Biointerfaces 2013, 103, 59-66.

41. Hu, H.; Larson, R. G., Analysis of the microfluid flow in an evaporating sessile droplet. Langmuir 2005, 21 (9), 3963-3971. 
42. Hu, H.; Larson, R. G., Analysis of the effects of Marangoni stresses on the microflow in an evaporating sessile droplet. Langmuir 2005, 21 (9), 3972-3980.

43. Majumder, M.; Rendall, C. S.; Eukel, J. A.; Wang, J. Y.; Behabtu, N.; Pint, C. L.; Liu, T. Y.; Orbaek, A. W.; Mirri, F.; Nam, J.; Barron, A. R.; Hauge, R. H.; Schmidt, H. K.; Pasquali, M., Overcoming the "coffee-stain" effect by compositional Marangoni-flow-assisted drop-drying. J Phys Chem $B$ 2012, 116 (22), 6536-42.

44. Cai, Y.; Zhang Newby, B.-m., Marangoni flow-induced self-assembly of hexagonal and stripelike nanoparticle patterns. Journal of the American Chemical Society 2008, 130 (19), 6076-6077. 


\title{
Self-Assembled Micro-stripe Patterning of Sessile Polymeric Nanofluid Droplets
}

\author{
Merve Gultekinoglu ${ }^{1,2}$, Xinyue Jiang ${ }^{3}$, Cem Bayram ${ }^{4}$, Han $\mathrm{Wu}^{5}$, \\ Kezban Ulubayram ${ }^{1,2,6,7}$, Mohan Edirisinghe ${ }^{3 *}$
}

${ }^{1}$ Department of Basic Pharmaceutical Sciences, Faculty of Pharmacy, Hacettepe University, Turkey

${ }^{2}$ Bioengineering Division, Institute for Graduate Studies in Science \& Engineering, Hacettepe University, Turkey

${ }^{3}$ Department of Mechanical Engineering, University College London (UCL), London WC1E 7JE, UK

${ }^{4}$ Advanced Technologies Application and Research Center, Hacettepe University, Ankara, Turkey

${ }^{5}$ Department of Chemical Engineering, University College London (UCL), London, WC1E 7JE, UK

${ }^{6}$ Nanotechnology \& Nanomedicine Division, Institute for Graduate Studies in Science \& Engineering, Hacettepe University, Turkey

${ }^{7}$ Polymer Science and Technology Division, Institute for Graduate Studies in Science \& Engineering, Hacettepe University, Turkey

* Corresponding author ME- m.edirisinghe@ucl.ac.uk 


\section{Abstract}

When sessile nanofluid droplets evaporate, solid nanoparticles can be organized in a wide variety of patterns on the substrate. The composition of the nanofluid, internal flow type of droplet and the rate of drying affect drop geometry, and the final pattern. Using poly(lactic-co-glycolic acid)-block-poly(ethylene glycol)(PLGA-b-PEG) as the example, we produced micro-stripe patterning from nanoparticles by drying of sessile fluid droplets. We investigated the nanoparticle properties and flow dynamics to clarify their effects on the patterning. Nanoparticles were prepared by hydrodynamic flow focusing using a T-junction microfluidic device with high production efficiency and the ability to generate an extremely narrow size distribution. PLGA-b-PEG was prepared as oil phase in acetonitrile and water/oil flow rate was changed from 1 to 3 at constant oil phase flow rate $(50 \mu \mathrm{L} / \mathrm{min})$. Then, nanofluid was collected on the surface as sessile droplets within acetonitrile/water binary dispersed phase. Depending on size, charge and size-distribution, the nanoparticles deposited on the surface exhibited various patterns. Dynamic Light and X-ray Scattering measurements showed that, approximately $100 \mathrm{~nm}$ particles with relatively low PDI (0.04) were produced for the first time in surfactant free conditions in a microfluidic device and they generated self-assembled ordered patterns, which are regulated by the type of internal flow in the sessile nanofluid droplet during sequential evaporation of acetonitrile and water.

Keywords: Nanofluid, hydrodynamic flow focusing, coffee-ring, self-assembly. 


\section{Introduction}

Nanofluid is basically defined as a colloidal suspension that contains nano-sized particles (nanoparticle-fluid suspensions). The suspended particles can be composed of metal, carbon or polymeric materials with various forms such as spherical nanoparticles ${ }^{1}$, carbon nanotubes ${ }^{2}$, nanowires ${ }^{3}$, nanofibers ${ }^{4}$ etc. Nanofluids generate uniform/non-uniform patterns when a sessile drop of suspension dries on a collecting layer. The sessile drops of nanofluids have an internal flow during the drying period and the pattern structure depends on this internal flow type. Moreover, nanoparticle size, shape, polydispersity, charge and material type directly affects the flow type and the pattern structure ${ }^{5-6}$. In addition, nanofluids have different evaporation, flow and spreading dynamics in contrast to pure solutions. In a sessile droplet of nanofluid, there are two main internal flow types, which are defined as Marangoni flow and Capillary flow.

A drying liquid droplet containing suspended solid particles leaves a ring-shaped trace along the boundary. This phenomenon is called coffee ring structure and can be encountered in daily life, such as stains left from coffee spills ${ }^{7}$. The scientific explanation of the coffee ring formation is based on fluid dynamics reported by Deegan et al. ${ }^{8}$ for the first time. The coffee ring formation is mainly caused by the solvent evaporation at the edge of the droplets. The edges are refilled with solvent redrawn from the centre and the solute carried by the flow also deposits at the edge, leaving a ring structure formation caused by particle deposition. However, in clean droplet interfaces without any surfactants, thermal Marangoni flow, which is caused by the evaporation, is also dominant in coffee ring structure formation on the centre of the droplet, rather than at the edges ${ }^{9}$. Basically, capillary flow induces uniform 
coffee-ring deposition, concentric rings and central deposition are induced by

Marangoni flow. In addition to elucidation of both, Sangani et al. ${ }^{10}$ reported that contact line of the coffee ring also depends on suspended particle size and concentration. These authors stated that the particles drawn to the edge may prevent the drop edge from receding during evaporation and cause more deposit formation. Revealing the transport phenomena of nanoparticles in evaporating droplets will lead to essential applications in lithography ${ }^{11}$, nanoparticle based coating $^{12}$, self-assembled patterns ${ }^{13}$ and bioseparation systems ${ }^{14}$.

For producing nanofluids which contains nanoparticles with small and narrow size distribution, homogeneous reaction and mixing conditions are crucial. Commonly, diminution of the device linear dimension, increase of temperature and concentration gradients are vital. Whitesides ${ }^{15}$ also stated that the microfluidic technologies have a pivotal role in monodisperse bubble, particle or droplet production due to controllable multiphase flow capabilities. Since microfluidic devices can supply accurate manipulation of mixing, residence time and reaction temperature, it has evolved into the most promising approaches to construct an effective mixing environment for nanoparticle preparation.

In contrast to conventional nanofluidic production methods, in the present study a precisely tuneable flow-focusing nanoprecipitation channel has been successfully created through a ready $\mathrm{T}$-junction micro-channel. In the literature, microfluidic technology was adapted for nanoparticle synthesis with microchip fluidic systems and there are several examples ${ }^{16-20}$. For instance, Valencia et al. ${ }^{16}$ performed nanoparticle synthesis with the aid of flow focusing in several microfluidic studies. In their work, polydimethylsiloxane (PDMS) micro-moulded channels were prepared and the main stream enabled flow of a polymeric solution, then they fed water phase from 
two parallel inlets and created the flow focusing to precipitate the nanoparticles. In our case, we chose the main flow stream as water and fed the polymer solution via lateral inlet of a $\mathrm{T}$-junction with a $90^{\circ}$ angle to the water stream. The polymer solution cut the water stream on the junction point of microfluidic device and feeding the polymer solution with controlled speed and concentration removes the need for surfactant in the synthesis. This is the very first design to make good use of laminar flow with low Reynolds number inside a microfluidic T-junction to produce a soft boundary co-axial nanoscale channel for one-step and surfactant free synthesis of nanoparticles. In contrast to traditional microfluidics, nanoparticles have been directly formed along the soft boundary co-axial channel, instead of forming microbubbles by immiscible solvents and burst to produce nanoparticles from their shells ${ }^{21}$. In this paper, we aimed to prepare self-assembled layers from concentrated nanofluids using PLGA-b-PEG polymeric nanoparticles with extremely low polydispersity index as an example, where layers are produced with the aid of our Tjunction microfluidic nanoparticle generation technique. For this purpose, PLGA-bPEG nanoparticles were synthesized in a $\mathrm{T}$-junction microfluidic device via hydrodynamic flow focusing. Then, self-assembled and highly ordered micro-stripe patterns were obtained according to the physicochemical characteristics of the nanofluids. Obtained pattern structures were characterized for size, charge, morphology and orientation. The effect of deposition parameters on the pattern structure is discussed in detail.

\section{Experimental section}

Materials: PLGA-b-PEG, 50:50, Resomer RGP d50105, d50155 (Mw=50000 g/mol) (diblock, 10\% and 15\% PEG) was obtained from Boehringer Ingelheim Pharma GmbH\&Co Ingelheim, Germany. Acetonitrile (anhydrous, 99.8\%) was obtained from 
Sigma-Aldrich, Germany. Polydimethylsiloxane (PDMS) microfluidic device (Tjunction) set up was fabricated by CNC machine in the University College London (UCL) Mechanical Engineering workshop, UK.

PLGA-b-PEG Polymer Solution Preparation and Characterization: PLGA-b-PEG polymer solution was prepared at 2.5, 5 and $10 \%(\mathrm{w} / \mathrm{v})$ concentrations in acetonitrile. Solution were magnetically stirred for $\sim 2$ hours prior to usage. It was not possible to process any higher concentration as their higher viscosity caused frequent blockage in the T-junction.

Nanofluid Production via T-junction Microfluidic Device: Microfluidic T-junction device assembly is described in detail in our previous work ${ }^{22-23}$. T-junction microfluidic device and nanofluid production by hydrodynamic flow focusing inside the junction are schematically represented in the Figure 1. Herein, two miscible phases were both fed by syringe pumps (Harvard Apparatus Ltd., Edenbridge UK) through FEP (Fluorinated Ethylene Polypropylene) tubing (both inner diameters at $200 \mu \mathrm{m}$ ) into a polydimethylsiloxane (PDMS) block. Another FEP capillary tubing (inner diameter $=200 \mu \mathrm{m}$ ) was fixed into the PDMS block as an outlet of the Tjunction. Water was fed into the junction from $y$-axis and oil phase (PLGA-b-PEG dissolved in acetonitrile) was fed from $\mathrm{x}$-axis (Figure 1A). While the oil phase flow rate was kept constant at $50 \mu \mathrm{L} / \mathrm{min}$ throughout the study, the water flow rates of 50 $\mu \mathrm{L} / \mathrm{min}, 100 \mu \mathrm{L} / \mathrm{min}$ and $150 \mu \mathrm{L} / \mathrm{min}$ were evaluated. Owing to the miscibility of solvents, acetonitrile and water, an overlapping zone was created in the middle of outlet tubing. A phantom 7.3 high speed camera with a maximum resolution of $800 \times 600$ pixels at up to $4800 \mathrm{fps}$ giving a recording duration of 1.2 seconds (Vision Research Ltd., UK) was utilized to document formation of the hydrodynamic flow focusing inside the T-junction channel. This overlapping zone also formed a soft 
boundary co-axial nanoscale channel inside the outlet tubing, where PLGA-b-PEG nanoparticles were later precipitated. Precipitated nanoparticles were collected using glass slides from the end of outlet tubing of the microfluidic T-junction as shown in Figure 1B. Sessile nanofluid droplet drying and the micro-stripe pattern formation are shown by schematic representation (Figure 1C) and the optical microscope images (Figure 1D). A video showing the process in action is given in Supplementary Information. PLGA-b-PEG polymer has a glass transition temperature $(\mathrm{Tg})$ of $\sim 41-43$ ${ }^{\circ} \mathrm{C}$, and therefore droplet drying procedure was carried out starting from ambient conditions up to $40{ }^{\circ} \mathrm{C}$. However, the studies were continued at room temperature since increasing temperature did not have shown significant effect on the obtained patterns.

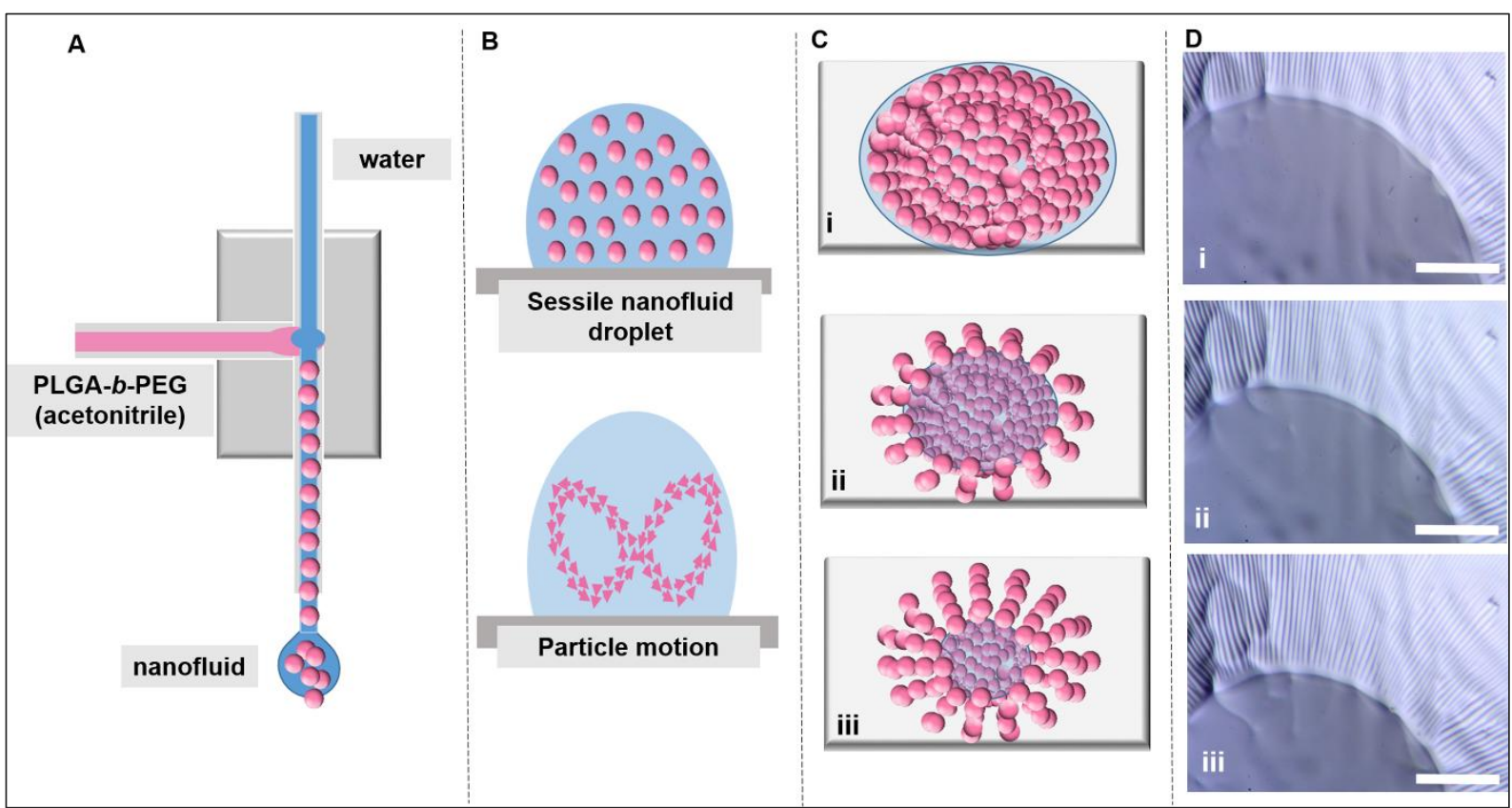

Figure 1. A) Schematic representation of PLGA-b-PEG nanoparticle based nanofluid production via hydrodynamic flow focusing, B) representative cross-sectional images of sessile nanofluid drop collection on glass slide and the particles motion inside the sessile droplet, C) representative image of i) sessile droplet, ii) drying and microstripe formation from orientated nanoparticles, iii) increased droplet drying and selfassembled pattern, D) optical microscope images of sessile droplet drying and microstripe formation, i-iii) shown step by step drying, scale bar $=200 \mu \mathrm{m}$. 
Characterization of Nanoparticles and Self Assembled Layers: Nanofluid (PLGAb-PEG nanoparticles dispersed in water-acetonitrile mixture) was characterized with small angle X-ray scattering (SAXS) and Dynamic Light Scattering (DLS) techniques (Malvern zetasizer ZS, UK). Nanoparticle size, zeta potential and polydispersity index were measured via DLS. Particle size was also determined on its native stage via SAXS. Nanoparticle crystallinity was checked by wide angle X-ray scattering (WAXS). SAXS/WAXS were performed using a GANESHA 300 XL SAXS/WAXS system equipped with a High Brilliance Micro focus source providing X-rays with a wavelength of $1.54 \AA$. A Pilatus $300 \mathrm{~K}$ solid-state photon-counting detector is located inside the vacuum chamber with full motorization. The change/movement between SAXS $(0.005 \leq q \leq 0.3)$ and WAXS $(2 \leq 2 \theta \leq 55)$ configuration can be achieved within 1 min to allow fast data collection $(q=4 \pi / \lambda(\sin \theta))$. Samples were contained in a custom designed glass cover transmission holder. Cost-friendly ACADEMY glass cover slips were used as background substrate.

Contact angle of nanofluid solution was measured by goniometer (Attension Theta, Biolin Scientific, Finland). Each sample contained an equal amount $(4 \mu \mathrm{L})$ of droplets collected on a glass slide. Contact angles were measured using an optical tensiometer (Attension Theta, Biolin Scientific, Finland) for 600 s to evaluate sessile drop drying motive and contact area evolution. Measurements were repeated three times for each sample. Morphological observations were performed with Optical Light Microscopy (Zeiss Axiotech, Germany with an attached Nikon Eclipse ME 600 camera, Japan), and Scanning Electron Microscopy (SEM, FEI Inspect S50, USA). Samples were coated with gold sputter prior to SEM analysis (Edwards, UK).

\section{Results and Discussion}


In the present study, nanofluids were produced by hydrodynamic flow focusing with serial concentrations of PLGA-b-PEG with $10 \%$ PEG content (PLGA-b-PEG 10 ) with acetonitrile as oil phase and PLGA-b-PEG nanoparticles were synthesized inside the microfluidic T-junction device. Then, PLGA-b-PEG nanoparticles were dried in a sessile droplet to generate self-assembled patterns. In the literature, most of the researchers focused on the self-assembling of nanoparticles with the aid of $\mathrm{pH}$, light, polarity, temperature, hydrophobicity, metallic interactions etc. ${ }^{24-26}$. Contrary to this, in this study we focused on the self-assembling patterns created by drying sessile droplets of polymeric nanoparticle suspensions. During nanoparticle generation, water and oil phases were fed into the $\mathrm{T}$-junction microfluidic device from different channels ( $x$ and $y$ directions) with different flow rates. Water phase was varied as 50 , 100 and $150 \mu \mathrm{L} / \mathrm{min}$ at constant flow rate of oil phase $(50 \mu \mathrm{L} / \mathrm{min})$. In the T-junction device, when water runs into the oil phase, PLGA-b-PEG precipitated directly in the water phase and nanoparticle formation occurred. It was found that PLGA-b-PEG concentration affected the nanoparticle properties, this was confirmed by both DLS and SAXS measurements (Table 1).

Table 1. The effect of PLGA- $b-P E_{10}$ (PEG 10 indicates PEG content in 10\%) concentration and the water/oil flow ratio in the microfluidic device on nanoparticle size (DLS and SAXS), PDI and zeta potential.

\begin{tabular}{cccccc}
$\begin{array}{c}\text { Polymer } \\
\%(\mathbf{w} / \mathbf{v})\end{array}$ & $\begin{array}{c}\text { Water/oil } \\
\text { Flow ratio }\end{array}$ & $\begin{array}{c}\text { Size } \\
\mathbf{D L S} \\
(\mathbf{n m})\end{array}$ & $\begin{array}{c}\text { Poly dispersity } \\
\text { index (PDI) }\end{array}$ & $\begin{array}{c}\text { Zeta } \\
\text { Potential } \\
(\mathbf{m V})\end{array}$ & $\begin{array}{c}\text { Size } \\
\text { SAXS } \\
(\mathbf{n m})\end{array}$ \\
\multirow{2}{*}{$\mathbf{2 . 5}$} & 1 & $140 \pm 1$ & $0.14 \pm 0.2$ & $-12.2 \pm 0.2$ & 141 \\
& 2 & $141 \pm 1$ & $0.13 \pm 0.1$ & $-8.1 \pm 0.4$ & 112 \\
& 3 & $140 \pm 1$ & $0.12 \pm 0.1$ & $-7.5 \pm 0.2$ & 128 \\
& 1 & $107 \pm 1$ & $0.06 \pm 0.01$ & $-8.5 \pm 0.4$ & 106 \\
$\mathbf{5}$ & 2 & $107 \pm 1$ & $0.05 \pm 0.01$ & $-8.5 \pm 0.5$ & 107 \\
& 3 & $108 \pm 1$ & $0.04 \pm 0.02$ & $-6.4 \pm 0.7$ & 109
\end{tabular}




$\begin{array}{lr}1 & 94 \pm 1 \\ 2 & 102 \pm 1 \\ 3 & 104 \pm 1\end{array}$

$0.15 \pm 0.2$

$-7.2 \pm 0.3$

From the DLS dataset collected under a constant flow ratio of $1: 1$, average nanoparticle size was determined as $140 \mathrm{~nm}$ for $2.5 \%$ PLGA-b-PEG 10 . It decreased to $107 \mathrm{~nm}$ for $5 \%$ PLGA- $b-\mathrm{PEG}_{10}$ and then further decreased to $94 \mathrm{~nm}$ for $10 \%$ PLGA- $b-P E G_{10}$. From the SAXS dataset of the same constant flow ratio, average nanoparticle size was determined as $141 \mathrm{~nm}$ for $2.5 \%$ PLGA- $b-P E G_{10}$, decreasing to

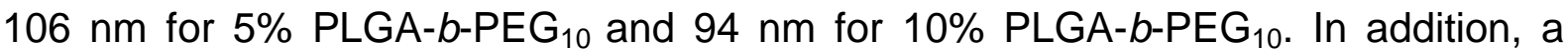
similar decreasing trend was observed at higher water/oil flow ratios which are also tabulated in Table 1. Average particle sizes were measured by DLS as $141 \mathrm{~nm}, 107$ $\mathrm{nm}$ and $102 \mathrm{~nm}$ at flow ratio 2 and $140 \mathrm{~nm}, 108 \mathrm{~nm}$ and $104 \mathrm{~nm}$ at flow ratio 3 for $2.5 \%, 5 \%$ and $10 \%$ PLGA- $b-\mathrm{PEG}_{10}$, respectively. Average particle sizes determined by SAXS were $112 \mathrm{~nm}, 107 \mathrm{~nm}$ and $94 \mathrm{~nm}$ at flow ratio 2 and $128 \mathrm{~nm}, 109 \mathrm{~nm}$ and $100 \mathrm{~nm}$ at flow rate ratio 3 for $2.5 \%, 5 \%$ and $10 \%$ PLGA- $b-\mathrm{PEG}_{10}$, respectively. Moreover, increasing polymer concentration caused an increase in zeta potential values. Zeta potential value was $-12.2 \mathrm{mV}$ for $2.5 \%$ PLGA- $b-P E G_{10},-8.5 \mathrm{mV}$ for $5 \%$ PLGA- $b-$ PEG $_{10}$ and $-7.2 \mathrm{mV}$ for $10 \%$ PLGA- $b-\mathrm{PEG}_{10}$ at a water/oil flow ratio of 1 (50:50 $\mu \mathrm{L} / \mathrm{min})$. It was concluded that increasing polymer concentration and water/oil ratio decreased size and increased zeta potential of nanoparticles (Figure 1A-B). Nanoparticle sizes measured by DLS in solution are larger than those measured by SAXS in the solid state (Figure 1C). This is likely to be caused by the continuous growth of nanoparticles in the liquid state caused by prolonged interaction time between PEG hydrophilic chains and water. Cheng et al. $^{27}$ also concluded that PLGA- $b-\mathrm{PEG}_{10}$ nanoparticle size varies at different polymer concentrations in organic 
solvent but at a fixed PLGA- $b-P \mathrm{G}_{10}$ concentration and water/oil phase, difference in

particle sizes are very slight.

It is also observed that obtained PLGA-b-PEG 10 nanoparticles are highly monodispersed, and the polydispersity index (PDI) ranges between $0.17-0.04$. In the literature, PLGA-b-PEG 10 nanoparticles PDI is generally around $0.20^{28-29}$. In our case, especially $5 \%$ PLGA-b-PEG 10 , highly monodisperse nanoparticles with $0.06,0.05$ and $0.04 \mathrm{PDI}$ values for 1,2 and 3 water/oil flow rates, respectively, were generated which is a very remarkable new result for the literature. It was concluded that, water/oil flow ratio did not exhibit a significant difference on nanoparticle size, PDI and zeta potential, polymer concentration of PLGA- $b-P_{E} G_{10}$ was the most affective factor.
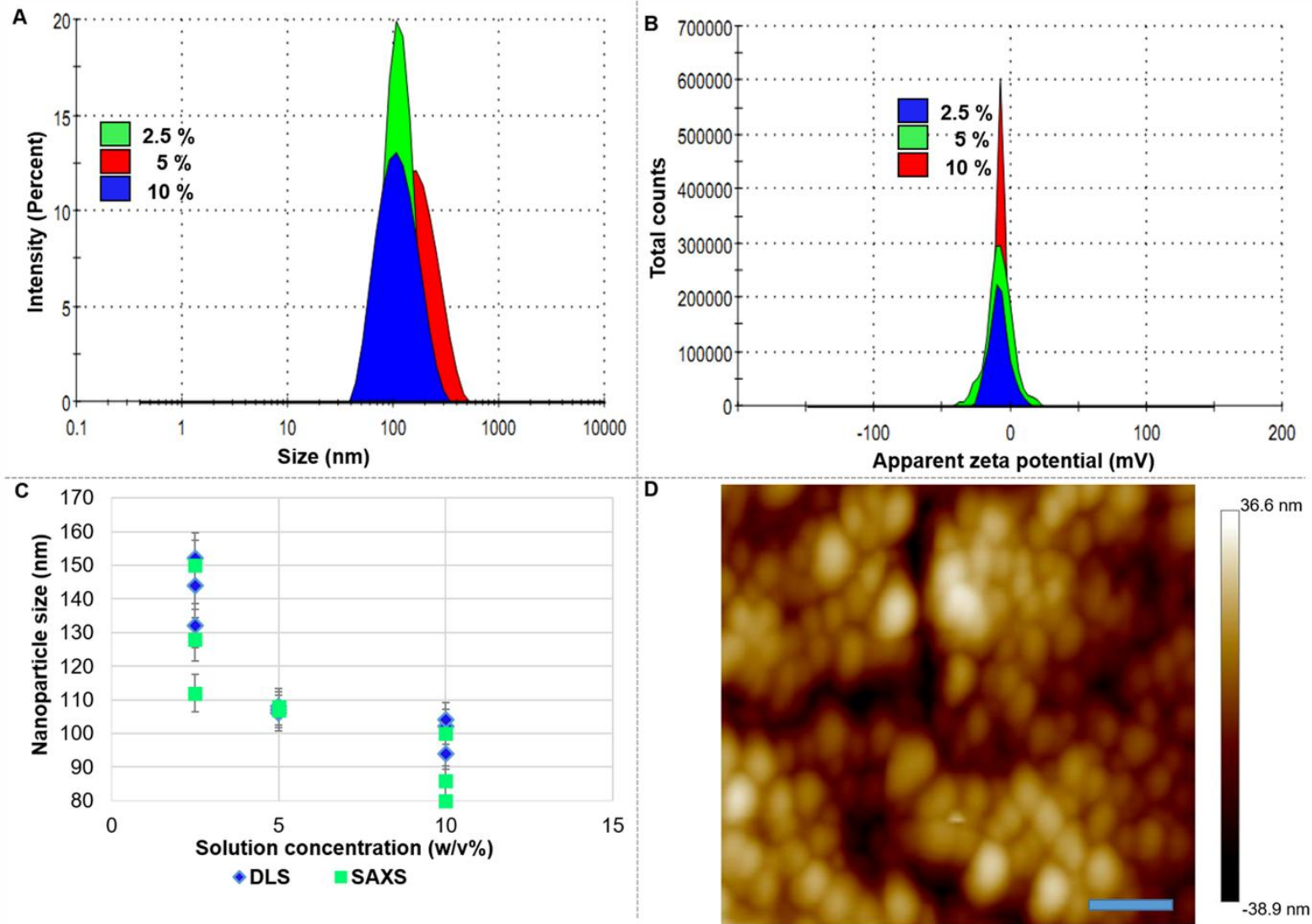

Figure 2. PLGA-b-PEG 10 nanoparticles $A$ ) average particle size $(n m)$, B) zeta potential $(\mathrm{mV})$ distribution graphs of $2.5 \%, 5 \%$ and $10 \%$ PLGA- $b$-PEG solutions (50:50 oil/water flow ratio), C) comparative size distribution graph of $2.5 \%, 5 \%$ and 
$10 \%$ PLGA- $b$-PEG solutions, D) AFM micrograph of PLGA- $b$-PEG nanoparticles, scale bar $=200 \mathrm{~nm}$ (50:50 oil/water flow ratio, $5 \%(\mathrm{w} / \mathrm{v})$ concentration).

Comparative size (Figure 2A) and zeta potential (Figure 2B) graphs represent the concentration gradient for $5 \%$ PLGA- $b-\mathrm{PEG}_{10}$ at flow ratio 1 . In addition, PLGA- $b$ $P E G_{10}$ nanoparticles size and morphology were also investigated by AFM. Figure 2D shows the AFM micrograph of highly monodisperse nanoparticles of $5 \%$ PLGA- $b$ $\mathrm{PEG}_{10}$ at flow ratio 1. AFM micrograph proved that the synthesized nanoparticles have spherical morphology and their size is around $100 \mathrm{~nm}$ diameter.



Figure 3. SAXS/WAXS measurements of PLGA- $b-P_{E G_{10}}$ nanoparticles formed in $5 \%$ $(w / v)$ solution at flow rate ratio 1 (oil:water) A) Log-log plot of SAXS pattern, B) Lin-lin plot of WAXS pattern shown in 2-theta configuration, C) full fit curve matched with data after subtracting background, D) 2D scattering pattern collected in SAXS, E) 2D scattering pattern collected in WAXS configuration.

It is known that results from SEM and DLS involve different kinds of post-synthesis operations, this may have a huge impact on the particle size. For this reason, we employed SAXS/WAXS technique with a specially-designed thin-film cell holder to examine the particles in their native state on the glass cover, following pattern 
formation and drying. No grinding, post-preparation or any disturbance was introduced to the sample prior to the SAXS measurements. We used a custom-made sample holder fitted with ACADEMY background cover slips to obtain structural information of the thin film nanoparticles in their native state. The difference between red curve (scattering from the sample) and blue curve (scattering from the blank glass cover, which is subtracted as the background) showed that that the noise-tosignal ratio is very low and therefore we could collect strong SAXS signals to reveal true features of the nanoparticles (Figure $3 \mathrm{~A}$ ). It proved that this cost-effective cover slides are very suitable substrates for nanoparticle thin films, also making them good alternatives to scatterless MICA/Kapton films which were widely used as window/background materials in SAXS measurements. Here, we used 'SAXSGUI' to do 'Full Fit' from the background subtracted data to obtain particle size information (Figure 3C). A polydispersed sphere model was used to fit the curve, and a Gaussian distribution was applied as it is known that the nanoparticles are spherical with some degree of polydispersity (5\% polydispersity from DLS measurement). The particle size determined by SAXS $(106 \mathrm{~nm})$ is smaller than those obtained from the DLS $(107 \mathrm{~nm})$ analysis. This very small difference is expected because DLS gives hydrodynamic radius which is known to be bigger than the so called 'true radius' measured from SAXS or microscopy. This indicated that in the process of the selfassembling and specific pattern formation, there is subtle change (or even no change) of these nanoparticle sizes from suspension state to solid state. The WAXS curve collected (Figure 3D) showed a typical amorphous scattering pattern with a very broad (amorphous) hump.

It is clear that PLGA-b-PEG nanoparticles remained amorphous during selfassembling and pattern formation processed. The observed high stability of this 
amorphous property is often key and preferred for optimising drug delivery, as unwanted crystallization or low bioavailability/dissolution rate can have a huge impact on drug safety, drug patent protection and other crucial issues in pharmaceutical industries. There have been several studies with forming polymeric, especially PLGA$b$-PEG nanoparticles ${ }^{30}$, nevertheless this is the first report studying sessile nanodroplet drying with self-assembled pattern structures of PLGA- $b$-PEG. SAXS/WAXS analyses showed that the particles retained their size and shape during the drying of sessile nanofluid droplets. Additionally, we investigated the sessile nanofluid droplets dried pattern structures. An additional data set with fixed flow ratio (1) and varying polymer concentrations (2.5, 5, $10 \%)$ was also studied to investigate the effect of PEG block content on the sessile droplet pattern, thus taking in to account PEG content, nanoparticle size, PDI and zeta potential. PLGA-b-PEG with $15 \%$ PEG content (PLGA-b-PEG ${ }_{15}$ ) exhibited higher hydrodynamic nanoparticle size with lower zeta potential values as a result of increasing PEG chain length. PDI values were also increased compare to values obtained from PLGA-b-PEG (Table 2).

Table 2. The effect of PEG content (15 in compare to 10 in Table 1) and polymer concentration on nanoparticles size, PDI and zeta potential.

$\begin{array}{ccccc}\begin{array}{c}\text { Polymer \% } \\ (\mathbf{w} / \mathbf{v})\end{array} & \begin{array}{c}\text { Water/oil } \\ \text { Flow ratio }\end{array} & \begin{array}{c}\text { Size } \\ \text { DLS } \\ (\mathbf{n m})\end{array} & \begin{array}{c}\text { Poly dispersity } \\ \text { index (PDI) }\end{array} & \begin{array}{c}\text { Zeta Potential } \\ (\mathbf{m V})\end{array} \\ \mathbf{2 . 5} & 1 & 175 \pm 1 & 0.16 \pm 0.1 & -1.9 \pm 0.1 \\ \mathbf{5} & 1 & 203 \pm 1 & 0.15 \pm 0.2 & -1.5 \pm 0.1 \\ \mathbf{1 0} & 1 & 266 \pm 2 & 0.24 \pm 0.1 & -0.8 \pm 0.2\end{array}$


An evaporating sessile droplet is characterized by its height, radius and contact angle values. Contact angle values of PLGA-b-PEG nanofluids were determined by sessile drop technique on a glass slide. Because of using a glass slide as the collector during the whole study it is important to determine and compare solution characteristics on the same surface. The evaporation mode of sessile droplet is classified by the change in one or some of these attributes during the drying process $^{31}$. As can be seen in Figure 3 the contact line is pinned in all PLGA-b-PEG 10 content keeping the radius of the contact area constant while a decrease in height is observed: in constant contact radius (CCR) mode, a linear contact angle decreases with linear volume loss. We believe that this two-step decreases are a result of a binary liquid system, occurring with the fast evaporation of volatile acetonitrile followed by water. Nevertheless, the evaporation of PLGA-b-PEG ${ }_{15}$ sessile droplets starts with the CCR mode, however it turns into constant contact angle (CCA) mode at 5 and 10 percentages as the radius decreases while the contact angle changes after 120s (Figure 4). 


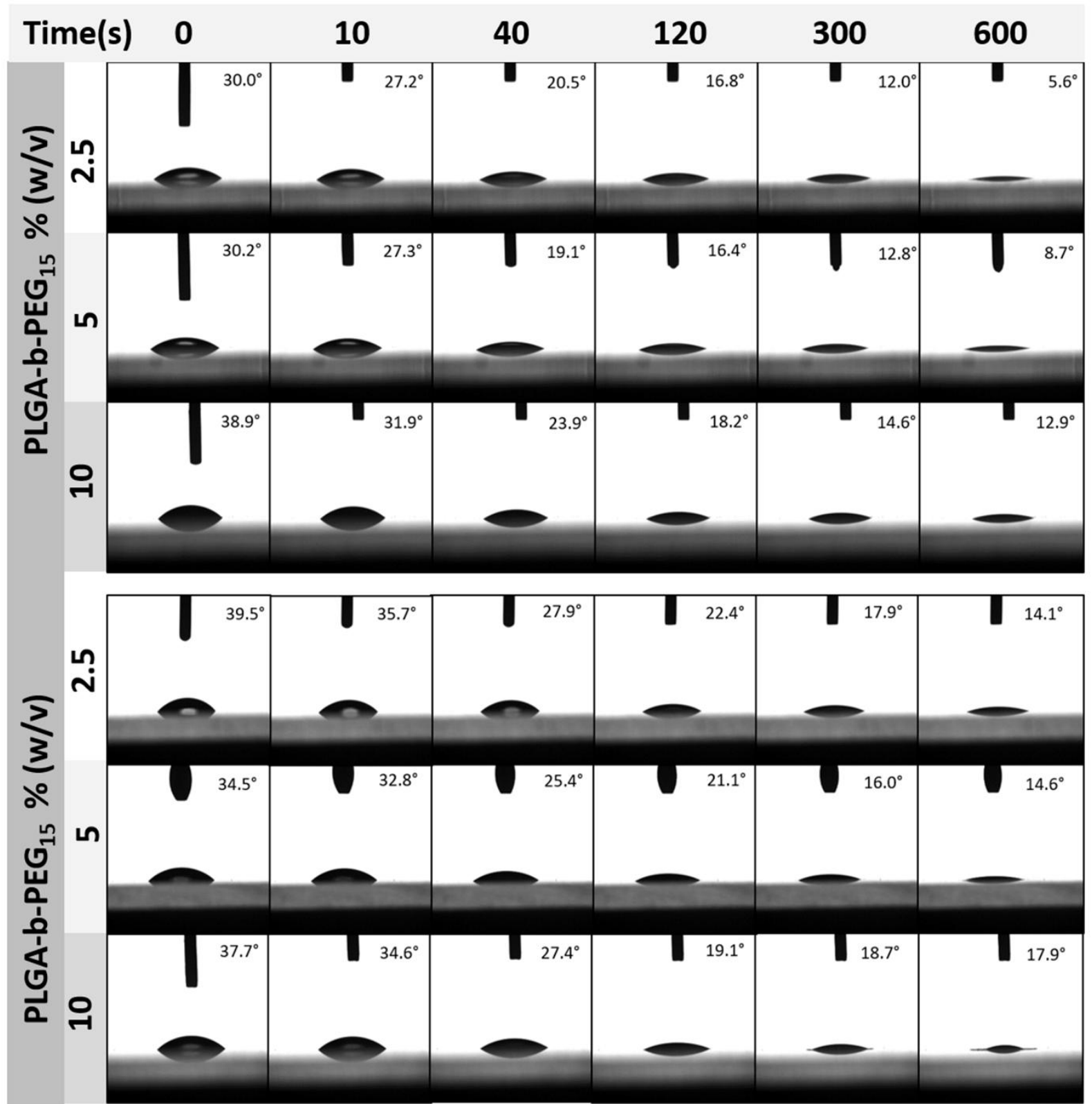

Figure 4. Contact angle values of PLGA- $b-P E G_{10}$ and PLGA- $b-P E G_{15}$ nanofluids of $2.5,5$ and $10 \%(\mathrm{w} / \mathrm{v})$ concentration at $\mathrm{t}=0,10,40,120,300,600 \mathrm{~s}$ of sessile droplet drying time periods.

The contact angle of PLGA- $b$-PEG 10 droplet is descending (Figure $5 \mathrm{~A}$ ) whereas, it remains the same in PLGA-b-PEG ${ }_{15}$ (Figure $5 B$ ). CCA phenomenon was previously reported by Erbil et al. ${ }^{32}$ with a diminishing drop height and area of contact. Small variations in contact angle values can be observed as McHale et al. ${ }^{33}$ reported, which is also seen in our study. However, they indicated that contact angle is a slow variable compared to area of contact. CCA angle mode is typically seen in smooth 
hydrophobic surfaces and the reason of this mode change can be caused by the high

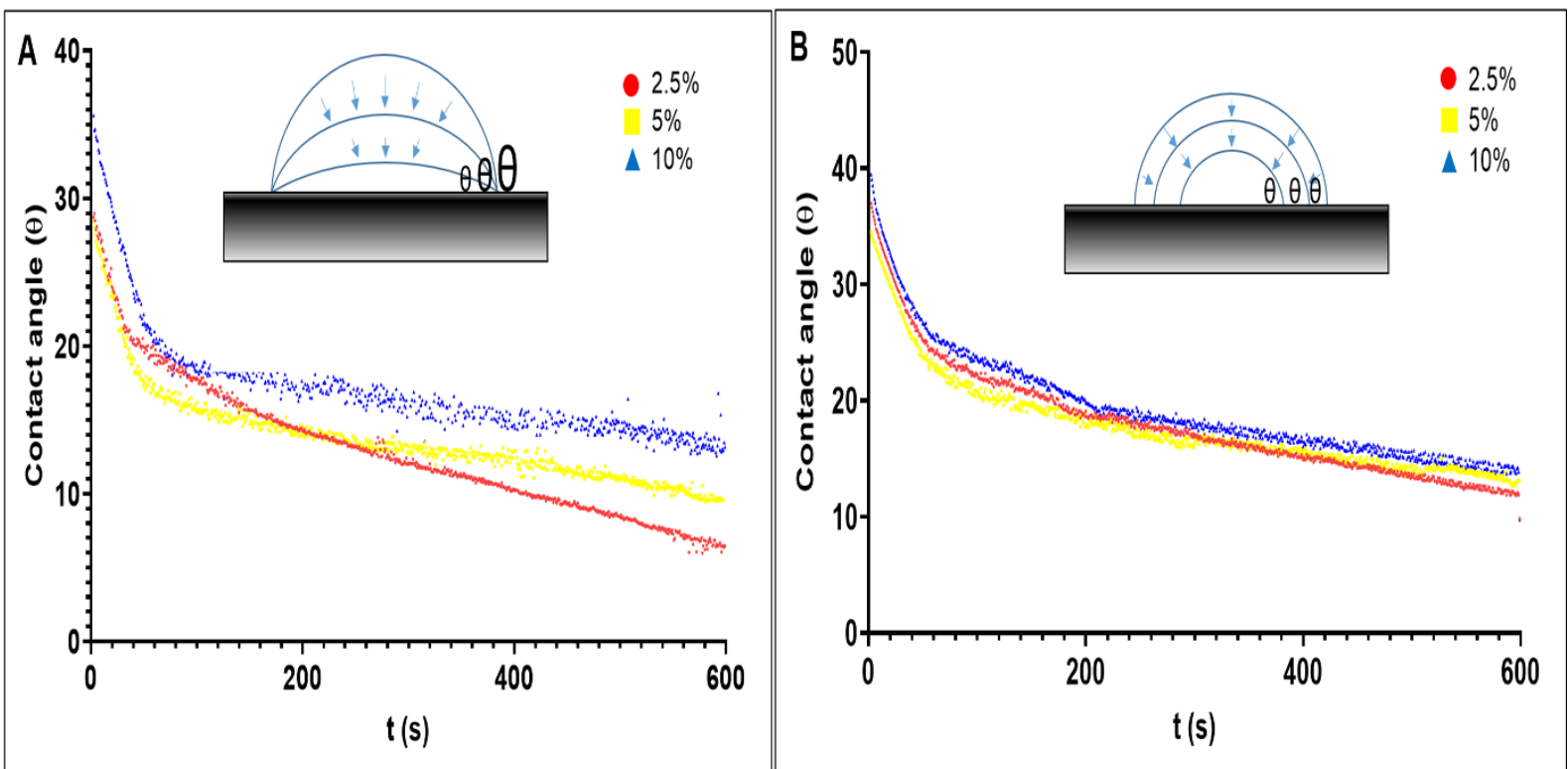

Figure 5. Contact angle versus time variation for 2.5, 5 and $10 \%(w / v)$ A) PLGA- $b$ $P G_{10}$ and $\left.B\right)$ PLGA- $b-P E G_{15}$ sessile droplet nanofluids.

CCA type evaporation mode indicates that at higher concentrations of PLGA-b$\mathrm{PEG}_{15}$ nanoparticles tend to deposit on the hydrophilic glass slide rather than move inside the droplet by internal flow, which can be interpreted by increasing hydrodynamic radius and neutralized zeta potentials compared to the same concentrations of PLGA-b-PEG 10 . Decreasing repelling forces and increased size of nanoparticles caused aggregation during the collision of the particles inside the droplet creating a hydrophobic layer. Vafaei et al. ${ }^{34}$ studied the effect of nanofluid concentration on surface wettability and stated that the contact angle of nanofluids increase with increasing concentration. Similarly, in our case contact angle of PLGA$b-P E G_{10}$ which have $C C R$ type contact angle increases with increasing concentration. 
The drying of nanofluid systems with CCR usually results with coffee stain patterns after the suppression of Marangoni effect ${ }^{35}$. However, uniform deposition of nanoparticles, especially at high densities, was also occasionally reported ${ }^{36-37}$. The denser population of nanoparticles with high viscosity suppresses the thermally induced Marangoni effect, resulting in a uniform thicker pattern in the contact area. Moreover, the contribution of faster evaporation on Marangoni effect is also stated in the literature; Bigioni et al. $^{38}$ reported an exceptional long range ordered nanoparticles by enhanced evaporation rate. Marangoni flow recirculates the nanoparticles inside the droplet, preventing them to deposit at the pinned edge. Concordantly, accumulation of nanoparticles at the air-liquid interface rather than drop edge is also possible by increasing the evaporation as reported by Li et al. ${ }^{1}$ who took into account that, the above mentioned examples include metallic and inorganic based nanofluids with single liquid systems. In our study, binary liquid system (wateracetonitrile) evaporates sequentially and with the aid of sequential drying motion the nanoparticles are evenly distributed inside the droplet caused by the Marangoni effect. 


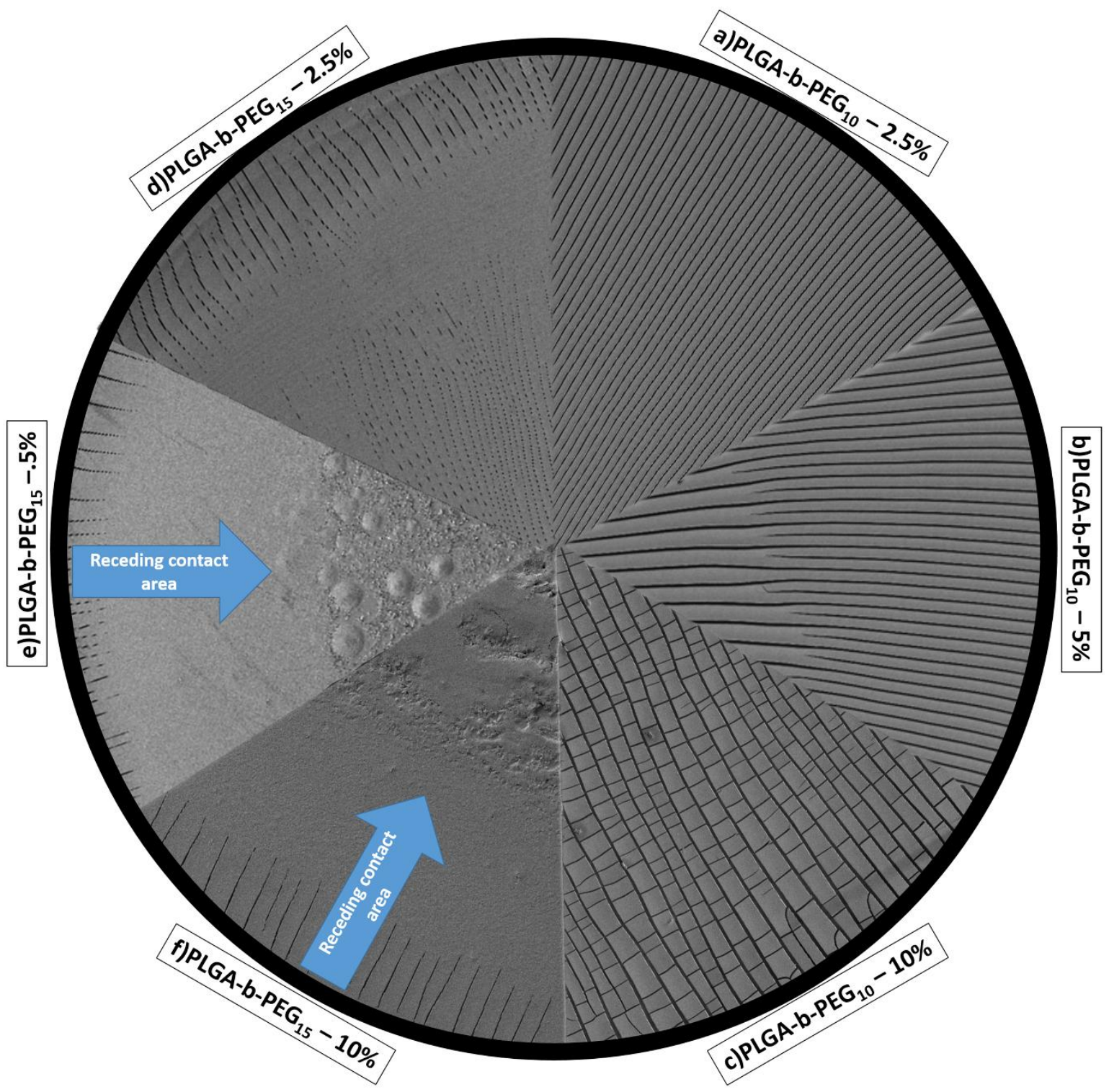

Figure 6. Scanning electron microscope images of self-assembled micro-stripe patterns from PLGA- $b$-PEG 10 and PLGA- $b$-PEG ${ }_{15}$ sessile nanofluid droplets produced at oil/water flow ratio 1 with different polymer concentrations. a) $2.5 \%$ b) $5 \%$ c) $10 \%$ (w/v) PLGA-b-PEG 10 and d) $2.5 \%$ e) 5\% f) $10 \%$ (w/v) PLGA-b-PEG ${ }_{15}$.

The sessile droplets dried at ambient conditions and different patterns were discovered for different PLGA- $b-\mathrm{PEG}_{10}$ and PLGA- $b-\mathrm{PEG}_{15}$ polymer concentrations. The obtained patterns were investigated by Scanning Electron Microscope (SEM) are summarised in Figure 6A-F. Evaporation of sessile nanofluid droplets is driven by two main flow regimes which are Capillary flow and Marangoni flow ${ }^{35,}$ 39. Here, the nanofluid droplet which was prepared from 2.5, 5 and 10\% (w/v) PLGA-b-PEG 10 
nanofluids created self-assembled micro-stripe patterns by highly ordered radial crack formation (Figure 5A-C). In Figure 5D-F, 2.5, 5 and 10\% (w/v) PLGA-b-PEG 15 nanofluid sessile droplet drying patterns were investigated and it is seen that the cracks at the drop edge started to form in a similar way but were interrupted or completely disappeared later on. The latter case was seen on 5 and $10 \%$ which has also CCA type drying pattern indicating that crack formation did not occur on deposited layer of nanoparticles with larger size and lower zeta potential.

The reason for crack formation at the droplet edge is a result of two competing forces, substrate adhesion and deposit shrinkage through evaporation. As the droplet shrinks while creating a local stress from the edge, the adhesion force between the droplet and substrate compresses the structure. Crack formation starts at the edge as a result of increased tensile strength. In our study, receding of contact area is followed by crack formation in the final stage of evaporation, which is remarkable and dominant particularly in PLGA-b-PEG 10 samples, towards the central regions. The direction of the cracks is similar at all polymer concentrations, however the width between the adjoining cracks (micro-stripes) increases with concentration.

The uniform micro-stripe formation was monitored by optical microscope in a time dependent manner in Figure 6 for $5 \%(\mathrm{w} / \mathrm{v})$ PLGA-b-PEG 10 samples. Stripes propagate continuously by $\sim 100 \mathrm{~nm}$ nanoparticles at $2 \mathrm{~s}$ intervals from Figure 7-A to 7-H. Stripes have uniform morphology in terms of both linearity and diameter with the assistance of Marangoni recirculation inside the sessile nanofluid droplet. Crack formation is observed by light microscopy as the wet front recedes and it causes an instability in the liquid zone followed by crack micro-stripe patterning. 
$\bar{E}$

$\bar{F}$

$\bar{G}$

Figure 7. Optical microscope images of $5 \%(\mathrm{w} / \mathrm{v})$ PLGA-b-PEG 10 sessile nanofluid droplets drying as a function of time, scale bar $=50 \mu \mathrm{m}(\mathrm{A}-\mathrm{H}$, time intervals $=2 \mathrm{~s}$, oil/water flow ratio 1 ).

Gorr et al..$^{40}$ stated that the width and the direction of the cracks depends on the drop shape evolution. In our experiments, we observed that drying sessile drops obeying the CCR type has well-ordered crack formation and propagation through the radial direction. The crack formation was also observed in PLGA-b-PEG ${ }_{15}$ sample groups, however the propagation in the radial direction continues only in $2.5 \%$ concentration with an interruption. Crack formation at the edges was also noticed at higher densities of PLGA-b-PEG ${ }_{15}$, though the crack propagation disappeared after the ring area indicating the tensile stress does not reach the local tensile strength. Additionally, multiple distinct bumps and irregular intrusions were also seen in 5 and $10 \%(\mathrm{w} / \mathrm{v})$ concentrations of PLGA-b-PEG ${ }_{15}$, respectively. Amongst the six patterns shown in Figure 6, nanoparticles created the most uniform, highly ordered and continuous micro-stripe patterns along the evaporation direction. In Figure $6 \mathrm{C}$ the tensile strength during evaporation is so high that orthoradial cracks were also observed in the obtained pattern. 
The uniform crack formations and the nanoparticles embedded in micro-stripes are shown in Figure 8A-F. Also, embedded nanoparticles were shown in Figures 8D-F where the nanoparticle size is confirmed by SEM, AFM, SAXS/WAX and DLS measurements as $\sim 100 \mathrm{~nm}$. Radial crack width increases with the nanoparticle concentration. This tendency can be explained by $2.5 \%(\mathrm{w} / \mathrm{v})$ PLGA-b-PEG 10 (Figure $8 \mathrm{~A}$ ) has the thinner uniform linear patterns than $5 \%(\mathrm{w} / \mathrm{v})$ PLGA-b-PEG 10 (Figure 8B) and $10 \%(w / v)$ PLGA-b-PEG 10 (Figure $8 C$ ) has linear patterns with orthoradial linear cracks since it has the highest polymer concentration (10\%) and the highest concentration of nanoparticles in an equal amount of sessile droplet amongst the samples. The width of micro-stripes was measured as $8.5 \pm 0.2,14.2 \pm 0.3$ and $14.8 \pm 0.3 \mu \mathrm{m}$ for $2.5,5$ and $10 \%$ (w/v) PLGA-b-PEG 10 , respectively (Figure $8 A-C$ ).

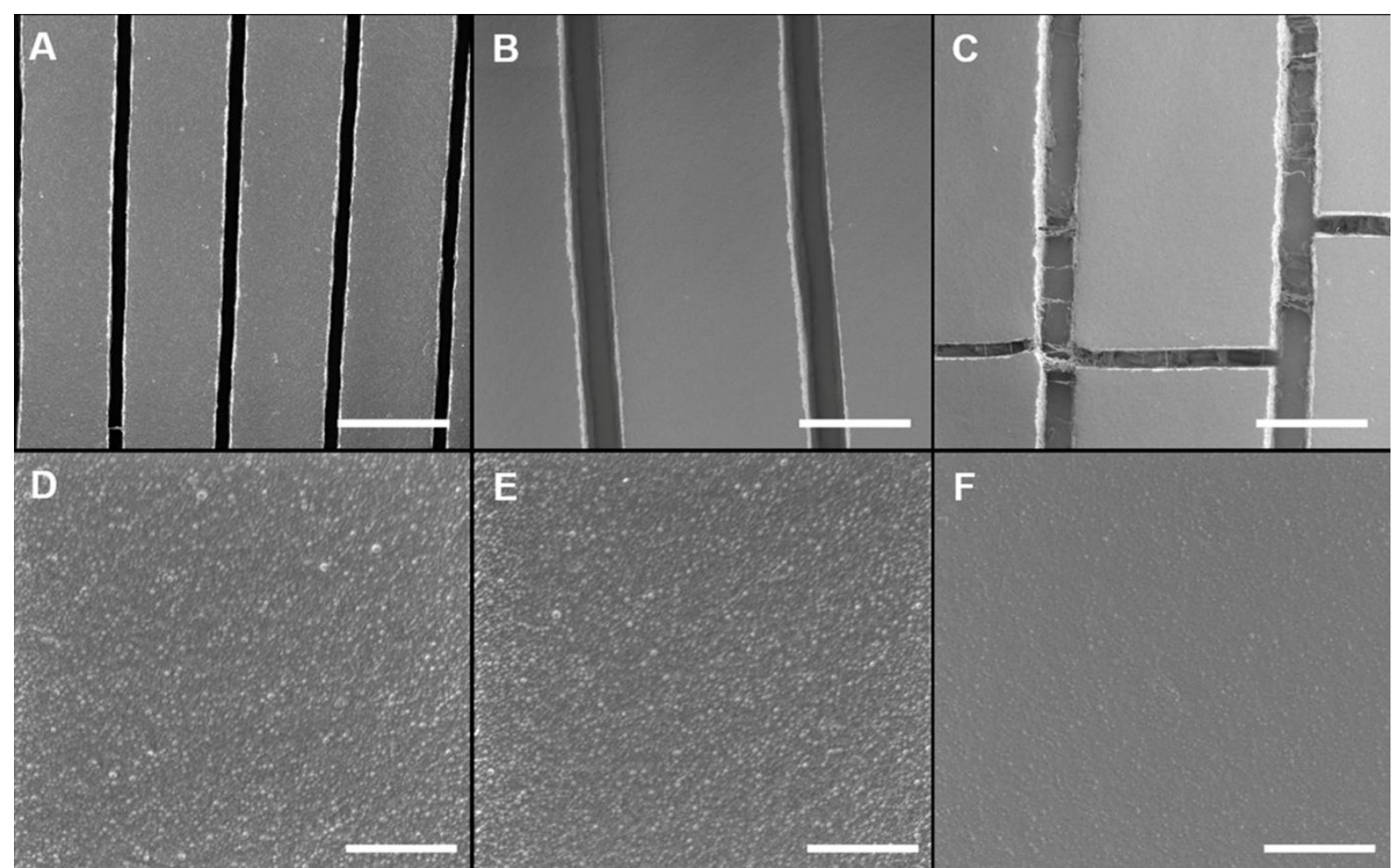

Figure 8. Scanning electron microscope images of self-assembled patterns from PLGA- $b-\mathrm{PEG}_{10}$ sessile nanofluid droplets produced at oil/water flow ratio 1 with different polymer concentrations A) 2.5, B) 5 , C) $10 \%$ (w/v) PLGA-b-PEG 10 (scale bar $=10 \mu \mathrm{m}$ ) and high magnification micrographs of embedded nanoparticles of $D$ ) 2.5 , E) 5, F) $10 \%$ (w/v) PLGA-b-PEG 10 (scale bar= $2 \mu \mathrm{m}$ ). 
Deegan et al. ${ }^{8}$ reported that coffee-ring deposition is forced by Capillary flow which starts from a pinned contact line. Moreover, $\mathrm{Hu}$ and Larson $^{9}$ explained that the Marangoni effect should be supressed to obtain coffee-ring deposits, because the Marangoni effect forces the creation of self-assembled highly ordered patterns ${ }^{41-42}$. Here, sessile drops of nanofluid solutions dried sequentially. Vapour pressure of acetonitrile is higher than water, hence acetonitrile evaporates first. Acetonitrile concentration becomes higher at the edge of sessile droplet and reduces the surface tension of the droplet. Marangoni flow was induced by the change in surface tension and recirculation occurred inside the sessile droplet. The Stokes equation (Equation 1) gives the relationship between the gravitational force, settling velocity, density, viscosity and particle size ${ }^{43}$.

$$
V S=\frac{2}{9} \frac{\left(\rho_{p}-\rho_{f}\right)}{\mu} g R^{2}
$$

where Vs is the settling velocity, $\rho_{p}$ is particle density, $\rho_{f}$ is the nanofluid density, $\mu$ is nanofluid dynamic viscosity, $g$ is gravity and $R$ is the radius of the nanoparticles.

Marangoni effect was suppressed partially for 2.5 and totally for $5,10 \%(w / v)$ PLGA$b$-PEG ${ }_{15}$ samples and uniform deposition was hindered. Besides, all concentrations of PLGA- $b-\mathrm{PEG}_{10}$ resulted in ordered micro-stripe patterning due to the Marangoni flow with the optimal particle size for self-assembly patterns. In the present study, stripe formation threshold was found to be $\sim 175 \mathrm{~nm}$ for PLGA- $b$-PEG nanoparticles whereas uniform and continuous formation occurred at $\sim 100 \mathrm{~nm}$. Moreover, effect of zeta potential on uniform micro-stripe formation is dominant. Cai et al. ${ }^{44}$ also reported that $100 \mathrm{~nm}$ polystyrene nanoparticles were assembled into stripe like patterns with Marangoni flow. This particle size threshold can be explained using Equation 1, 
because nanoparticle circulation velocity is proportional to the square of particle radius. Moreover, velocity and flow type was affected not only by particle size but also by density of nanoparticles and nanofluids. For instance, 5 and 10\% (w/v) PLGA-b-PEG have the same particle size and they both deposited as micro-stripe patterning. Besides, $5 \%$ samples have highly ordered micro-stripe patterns due to the lower particle and nanofluid density gradient (Equation 1). It was also concluded that highly monodisperse (relatively low PDI) and highly charged (zeta potential) particles deposited as thinner stripes (Figure 6A-B and Figure 8A-C).

\section{Conclusions}

In this study, highly monodisperse PLGA-b-PEG nanoparticles were obtained by surfactant free hydrodynamic flow focusing in a T-junction microfluidic device. Synthesized nanoparticles were collected as nanofluid solutions and the nanofluids were dried as sessile droplets. Due to the internal flow type of the sessile droplets different deposition patterns were obtained. Internal flow type and the related deposition patterns were affected by the polymer concentration, nanoparticles size, charge and polydispersity index. PLGA-b-PEG nanoparticles which have extremely low PDI and $100 \mathrm{~nm}$ particle size were deposited as highly uniform self-assembled parallel stripes. Thus, T-junction microfluidic devices are ideal systems for the surfactant free, highly monodisperse nanoparticle fabrication and these nanoparticles exhibit highly ordered and self-assembled stripe patterns under optimized conditions.

\section{Acknowledgements}

This study was financially supported by Hacettepe University, Scientific Research Projects Coordination Unit (Grant Numbers: TDK-2017-14725 and FBI-2017-14296). 


\section{References}

1. Li, C. H.; Peterson, G., Experimental investigation of temperature and volume fraction variations on the effective thermal conductivity of nanoparticle suspensions (nanofluids). Journal of Applied Physics 2006, 99 (8), 084314.

2. Xie, H.; Lee, H.; Youn, W.; Choi, M., Nanofluids containing multiwalled carbon nanotubes and their enhanced thermal conductivities. Journal of Applied Physics 2003, 94 (8), 4967-4971.

3. Fang, X.; Ding, Q.; Fan, L.-W.; Lu, H.; Yu, Z.-T., Effects of inclusion size on thermal conductivity and rheological behavior of ethylene glycol-based suspensions containing silver nanowires with various specific surface areas. International Journal of Heat and Mass Transfer 2015, 81, 554-562.

4. Lee, K. J.; Yoon, S. H.; Jang, J., Carbon nanofibers: a novel nanofiller for nanofluid applications. Small 2007, 3 (7), 1209-1213.

5. Shen, X.; Ho, C.-M.; Wong, T.-S., Minimal size of coffee ring structure. The Journal of Physical Chemistry B 2010, 114 (16), 5269-5274.

6. Ryu, S.-a.; Kim, J. Y.; Kim, S. Y.; Weon, B. M., Drying-mediated patterns in colloid-polymer suspensions. Scientific Reports 2017, 7 (1), 1079.

7. Han, W.; Lin, Z., Learning from "Coffee Rings": Ordered Structures Enabled by Controlled Evaporative Self-Assembly. Angewandte Chemie International Edition 2012, 51 (7), 1534-1546.

8. Deegan, R. D.; Bakajin, O.; Dupont, T. F.; Huber, G.; Nagel, S. R.; Witten, T. A., Capillary flow as the cause of ring stains from dried liquid drops. Nature 1997, 389 (6653), 827.

9. Hu, H.; Larson, R. G., Marangoni effect reverses coffee-ring depositions. The Journal of Physical Chemistry B 2006, 110 (14), 7090-7094.

10. Sangani, A. S.; Lu, C.; Su, K.; Schwarz, J. A., Capillary force on particles near a drop edge resting on a substrate and a criterion for contact line pinning. Physical Review E 2009, 80 (1), 011603.

11. Harris, D. J.; Conrad, J. C.; Lewis, J. A., Evaporative lithographic patterning of binary colloidal films. Philosophical Transactions of the Royal Society of London A: Mathematical, Physical and Engineering Sciences 2009, 367 (1909), 5157-5165.

12. Layani, M.; Gruchko, M.; Milo, O.; Balberg, I.; Azulay, D.; Magdassi, S., Transparent conductive coatings by printing coffee ring arrays obtained at room temperature. ACS nano 2009, 3 (11), 3537-3542.

13. Vohra, V.; Bolognesi, A.; Calzaferri, G.; Botta, C., Self-assembled nanofibers of fluorescent zeolite L crystals and conjugated polymer. Langmuir 2010, 26 (3), 1590-1593.

14. Smalyukh, I. I.; Zribi, O. V.; Butler, J. C.; Lavrentovich, O. D.; Wong, G. C., Structure and dynamics of liquid crystalline pattern formation in drying droplets of DNA. Physical Review Letters 2006, 96 (17), 177801.

15. Whitesides, G. M., The origins and the future of microfluidics. Nature 2006, 442 (7101), 368-

73.

16. Valencia, P. M.; Farokhzad, O. C.; Karnik, R.; Langer, R., Microfluidic technologies for accelerating the clinical translation of nanoparticles. Nature Nanotechnology 2012, 7 (10), 623.

17. Ali, H. S.; York, P.; Ali, A. M.; Blagden, N., Hydrocortisone nanosuspensions for ophthalmic delivery: a comparative study between microfluidic nanoprecipitation and wet milling. Journal of Controlled Release 2011, 149 (2), 175-181.

18. Liu, D.; Cito, S.; Zhang, Y.; Wang, C. F.; Sikanen, T. M.; Santos, H. A., A versatile and robust microfluidic platform toward high throughput synthesis of homogeneous nanoparticles with tunable properties. Advanced Materials 2015, 27 (14), 2298-2304.

19. Valencia, P. M.; Basto, P. A.; Zhang, L.; Rhee, M.; Langer, R.; Farokhzad, O. C.; Karnik, R., Single-step assembly of homogenous lipid- polymeric and lipid- quantum dot nanoparticles enabled by microfluidic rapid mixing. ACS nano 2010, 4 (3), 1671-1679.

20. Valencia, P. M.; Pridgen, E. M.; Rhee, M.; Langer, R.; Farokhzad, O. C.; Karnik, R., Microfluidic platform for combinatorial synthesis and optimization of targeted nanoparticles for cancer therapy. ACS nano 2013, 7 (12), 10671-10680. 
21. Elsayed, M.; Kothandaraman, A.; Edirisinghe, M.; Huang, J., Porous Polymeric Films from Microbubbles Generated Using a T-Junction Microfluidic Device. Langmuir 2016, 32 (50), 1337713385.

22. Gultekinoglu, M.; Jiang, X.; Bayram, C.; Ulubayram, K.; Edirisinghe, M., Honeycomb-like PLGAb-PEG Structure Creation with T-junction Micro Droplets. Langmuir 2018, 34 (27), 7989-7997.

23. Bayram, C.; Jiang, X.; Gultekinoglu, M.; Ozturk, S.; Ulubayram, K.; Edirisinghe, M. J. M. M.; Engineering, Biofabrication of Gelatin Tissue Scaffolds with Uniform Pore Size via Microbubble Assembly. Macromolecular Materials and Engineering 2019, 1900394.

24. Grzelczak, M.; Vermant, J.; Furst, E. M.; Liz-Marzán, L. M., Directed self-assembly of nanoparticles. ACS nano 2010, 4 (7), 3591-3605.

25. Chen, Y.; Wang, Z.; He, Y.; Yoon, Y. J.; Jung, J.; Zhang, G.; Lin, Z., Light-enabled reversible selfassembly and tunable optical properties of stable hairy nanoparticles. Proceedings of the National Academy of Sciences 2018, 201714748.

26. Sánchez-Iglesias, A.; Grzelczak, M.; Altantzis, T.; Goris, B.; Perez-Juste, J.; Bals, S.; Van Tendeloo, G.; Donaldson Jr, S. H.; Chmelka, B. F.; Israelachvili, J. N., Hydrophobic interactions modulate self-assembly of nanoparticles. ACS nano 2012, 6 (12), 11059-11065.

27. Cheng, J.; Teply, B. A.; Sherifi, I.; Sung, J.; Luther, G.; Gu, F. X.; Levy-Nissenbaum, E.; RadovicMoreno, A. F.; Langer, R.; Farokhzad, O. C., Formulation of functionalized PLGA-PEG nanoparticles for in vivo targeted drug delivery. Biomaterials 2007, 28 (5), 869-876.

28. Baysal, I.; Ucar, G.; Gultekinoglu, M.; Ulubayram, K.; Yabanoglu-Ciftci, S., Donepezil loaded PLGA-b-PEG nanoparticles: their ability to induce destabilization of amyloid fibrils and to cross blood brain barrier in vitro. Journal of Neural Transmission 2017, 124 (1), 33-45.

29. Sari, E.; Tunc-Sarisozen, Y.; Mutlu, H.; Shahbazi, R.; Ucar, G.; Ulubayram, K., ICAM-1 targeted catalase encapsulated PLGA-b-PEG nanoparticles against vascular oxidative stress. Journal of Microencapsulation 2015, 32 (7), 687-698.

30. Şimşek, S.; Eroğlu, H.; Kurum, B.; Ulubayram, K., Brain targeting of Atorvastatin loaded amphiphilic PLGA-b-PEG nanoparticles. Journal of Microencapsulation 2013, 30 (1), 10-20.

31. Zhang, X.; Wang, J.; Bao, L.; Dietrich, E.; van der Veen, R. C.; Peng, S.; Friend, J.; Zandvliet, H. J.; Yeo, L.; Lohse, D., Mixed mode of dissolving immersed nanodroplets at a solid-water interface. Soft Matter 2015, 11 (10), 1889-1900.

32. Erbil, H. Y.; McHale, G.; Newton, M., Drop evaporation on solid surfaces: constant contact angle mode. Langmuir 2002, 18 (7), 2636-2641.

33. McHale, G.; Rowan, S. M.; Newton, M.; Banerjee, M. K., Evaporation and the wetting of a low-energy solid surface. The Journal of Physical Chemistry B 1998, 102 (11), 1964-1967.

34. Vafaei, S.; Borca-Tasciuc, T.; Podowski, M.; Purkayastha, A.; Ramanath, G.; Ajayan, P., Effect of nanoparticles on sessile droplet contact angle. Nanotechnology 2006, 17 (10), 2523.

35. Parsa, M.; Harmand, S.; Sefiane, K., Mechanisms of pattern formation from dried sessile drops. Advances in Colloid and Interface Science 2018.

36. Sefiane, K., On the role of structural disjoining pressure and contact line pinning in critical heat flux enhancement during boiling of nanofluids. Applied Physics Letters 2006, 89 (4), 044106.

37. Giorgiutti-Dauphiné, F.; Pauchard, L., Drying drops. The European Physical Journal E 2018, 41 (3), 32 .

38. Bigioni, T. P.; Lin, X.-M.; Nguyen, T. T.; Corwin, E. I.; Witten, T. A.; Jaeger, H. M., Kinetically driven self assembly of highly ordered nanoparticle monolayers. Nature Materials 2006, 5 (4), 265270.

39. Karpitschka, S.; Liebig, F.; Riegler, H., Marangoni Contraction of Evaporating Sessile Droplets of Binary Mixtures. Langmuir 2017, 33 (19), 4682-4687.

40. Gorr, H. M.; Zueger, J. M.; McAdams, D. R.; Barnard, J. A., Salt-induced pattern formation in evaporating droplets of lysozyme solutions. Colloids and Surfaces B: Biointerfaces 2013, 103, 59-66.

41. Hu, H.; Larson, R. G., Analysis of the microfluid flow in an evaporating sessile droplet. Langmuir 2005, 21 (9), 3963-3971. 
42. Hu, H.; Larson, R. G., Analysis of the effects of Marangoni stresses on the microflow in an evaporating sessile droplet. Langmuir 2005, 21 (9), 3972-3980.

43. Majumder, M.; Rendall, C. S.; Eukel, J. A.; Wang, J. Y.; Behabtu, N.; Pint, C. L.; Liu, T. Y.; Orbaek, A. W.; Mirri, F.; Nam, J.; Barron, A. R.; Hauge, R. H.; Schmidt, H. K.; Pasquali, M., Overcoming the "coffee-stain" effect by compositional Marangoni-flow-assisted drop-drying. J Phys Chem B 2012, 116 (22), 6536-42.

44. Cai, Y.; Zhang Newby, B.-m., Marangoni flow-induced self-assembly of hexagonal and stripelike nanoparticle patterns. Journal of the American Chemical Society 2008, 130 (19), 6076-6077. 
A



B
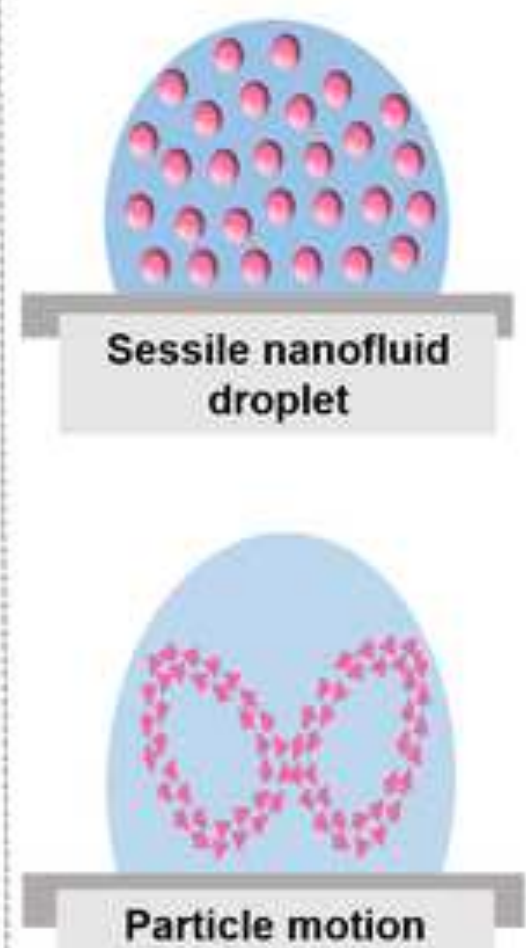

Particle motion

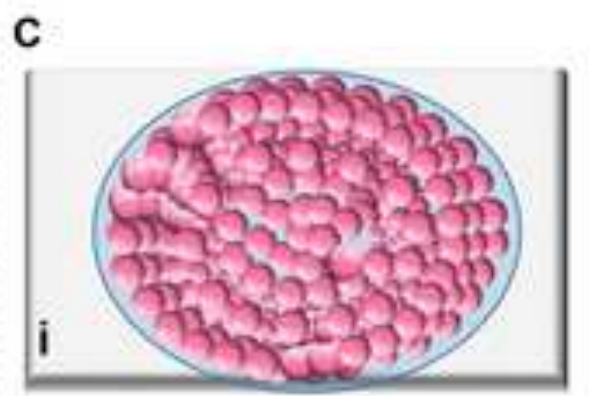

D
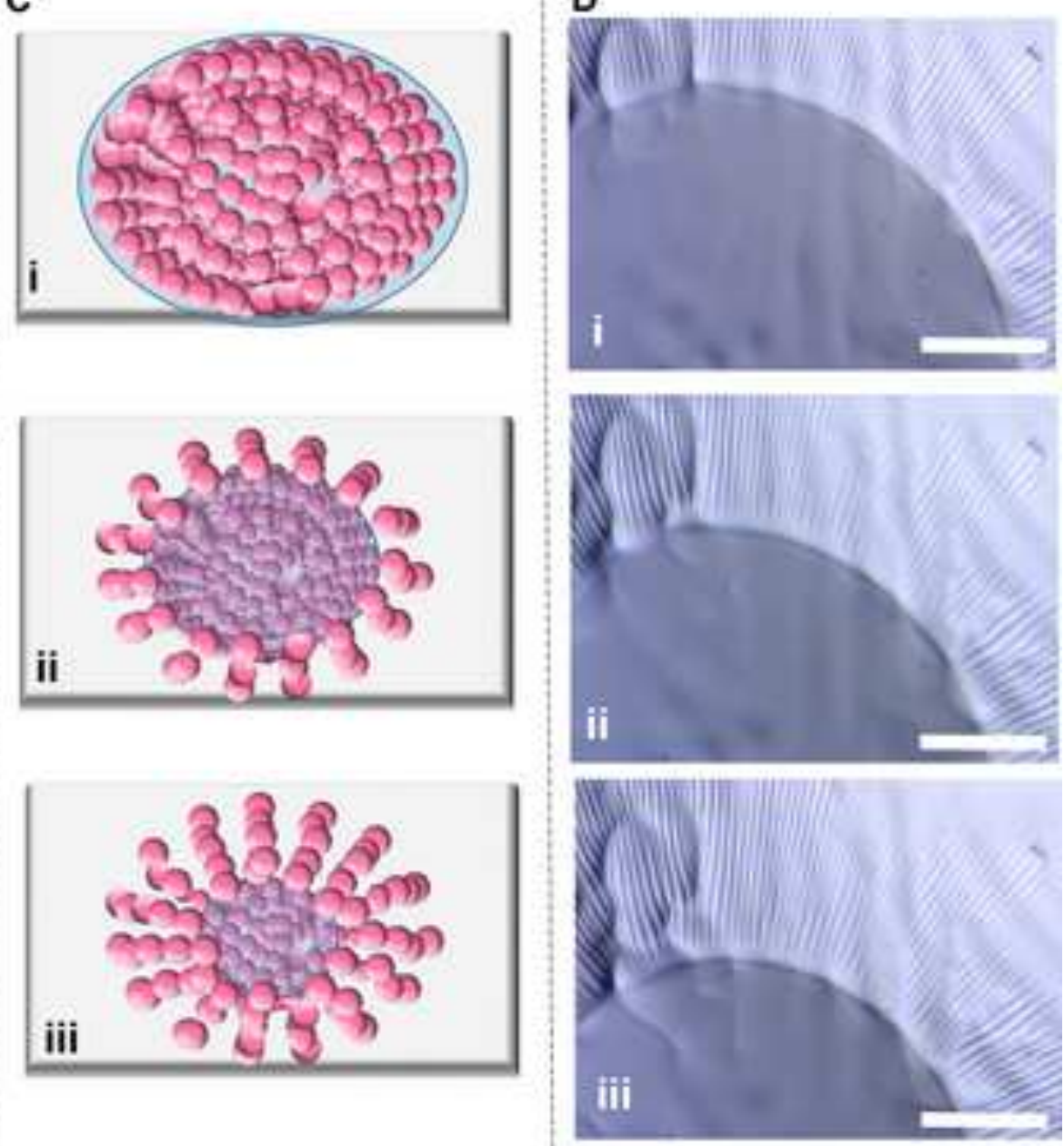
A
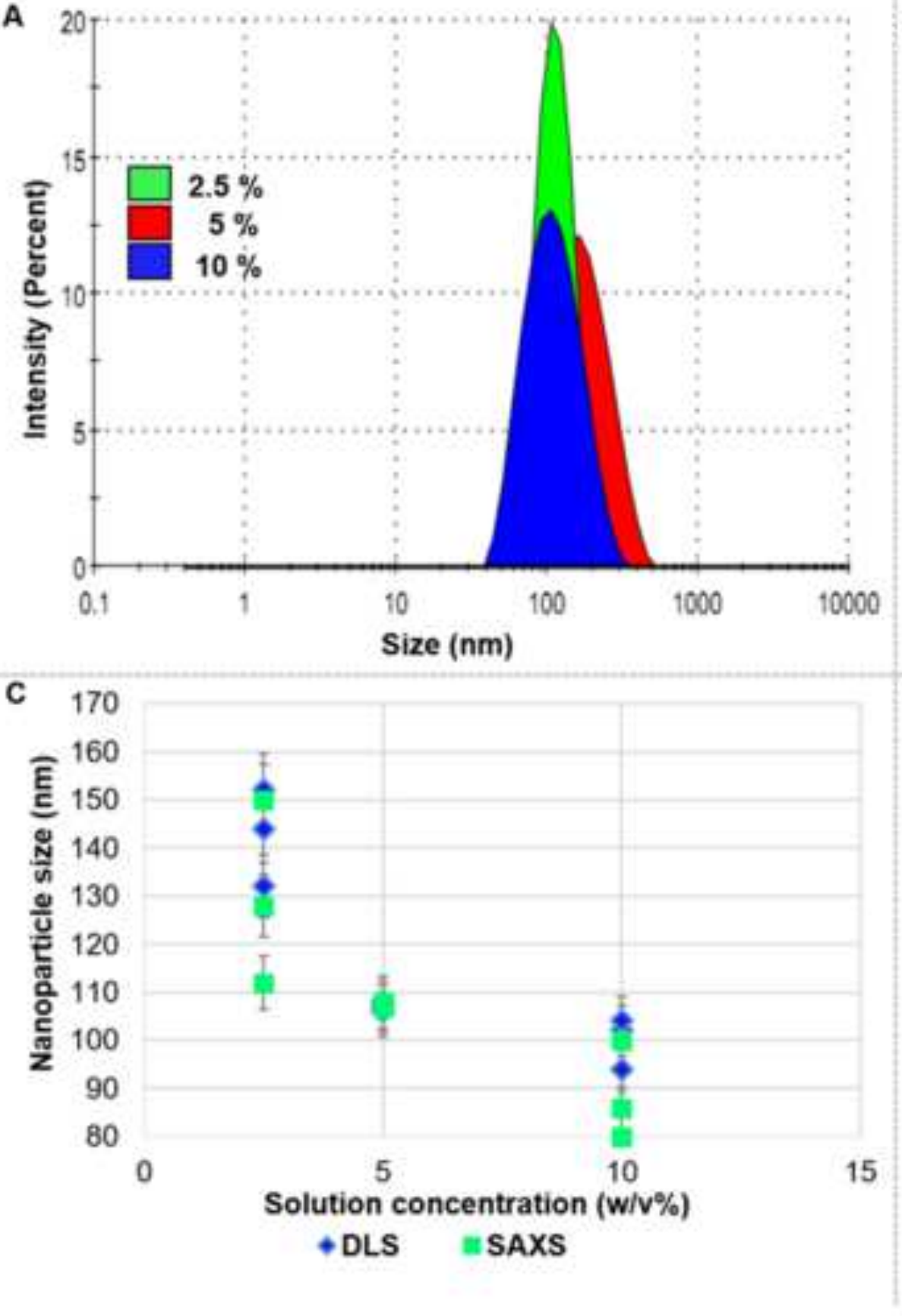

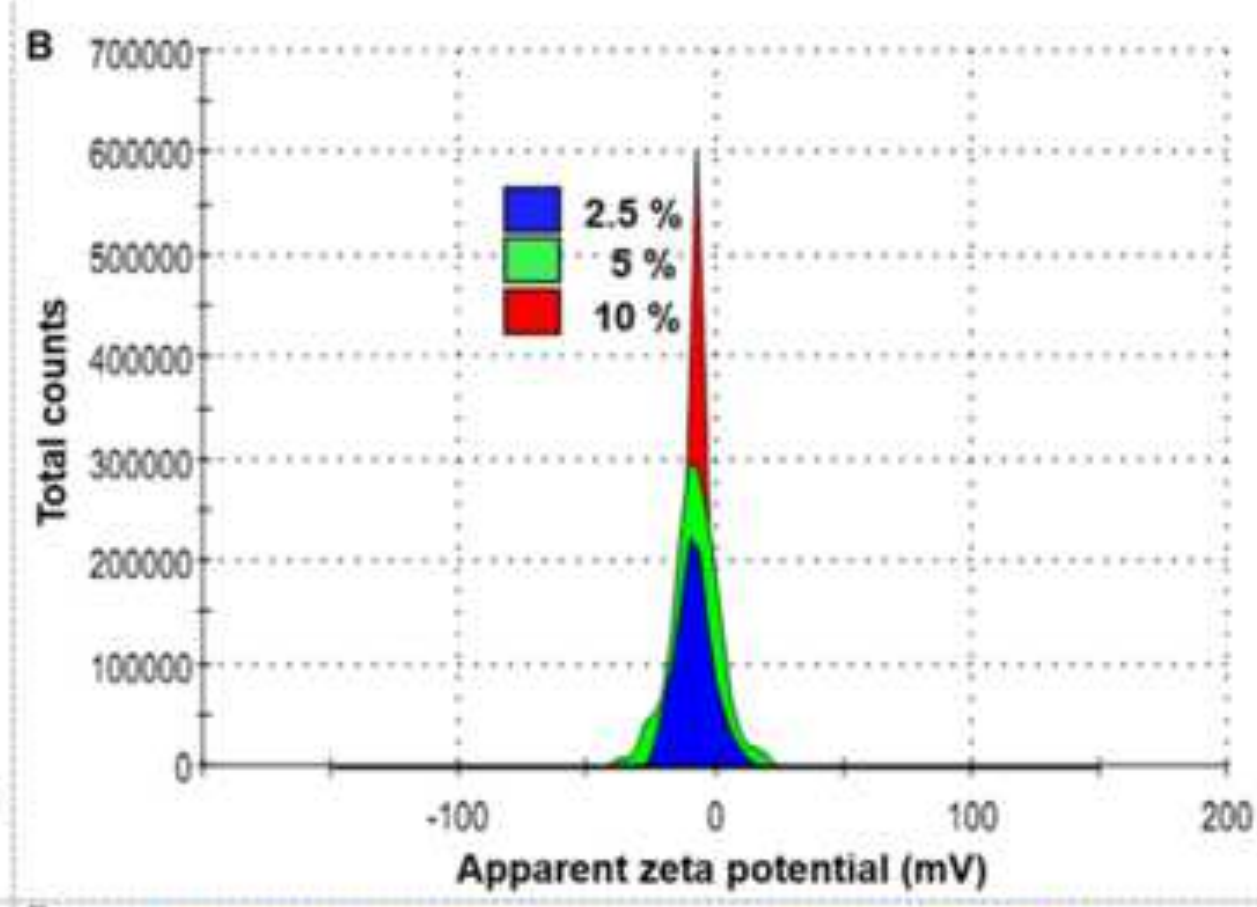

D

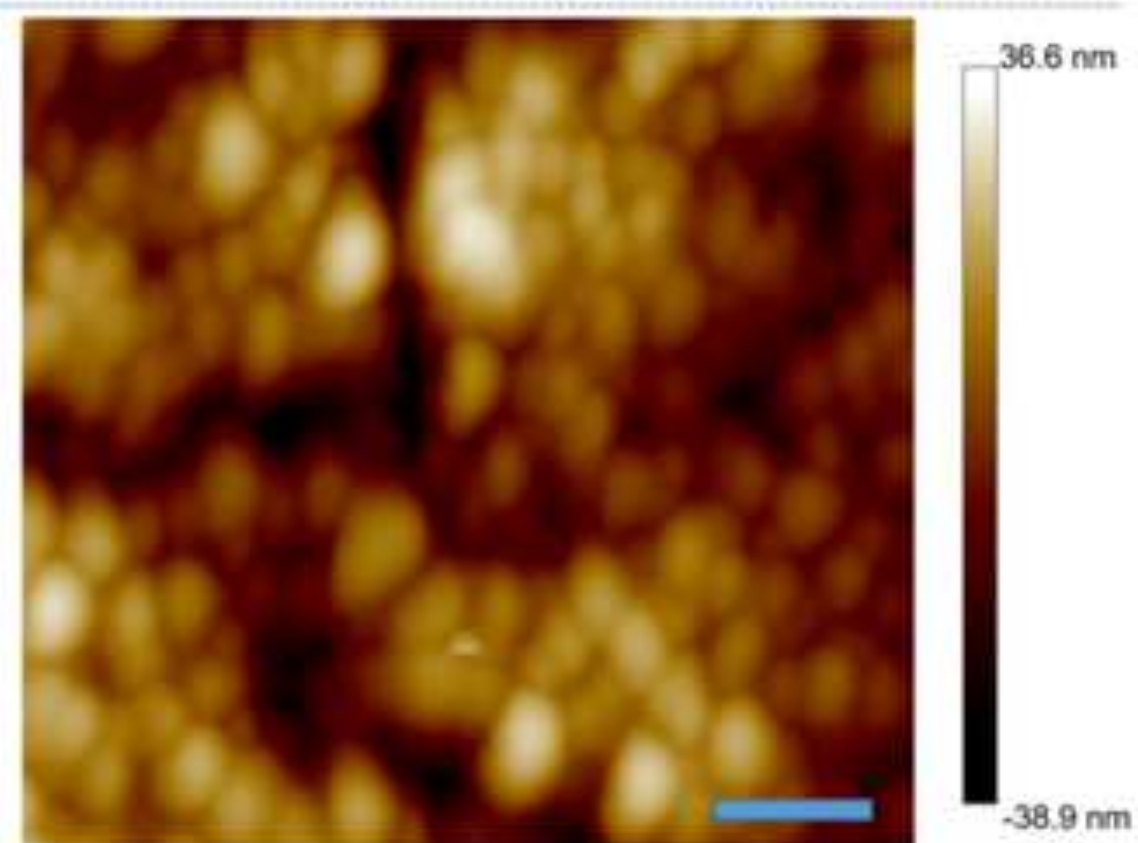




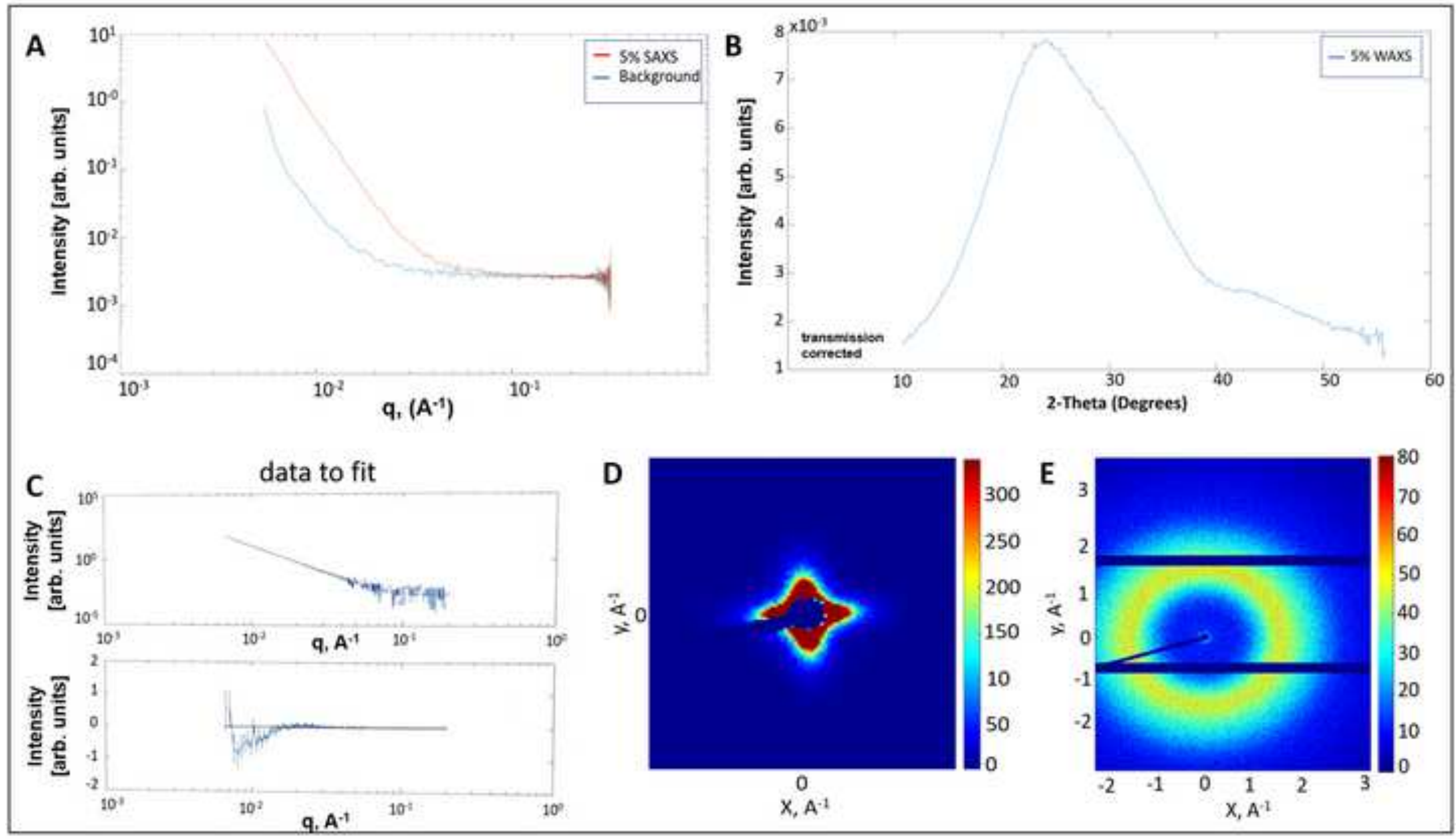







Click here to download high resolution image



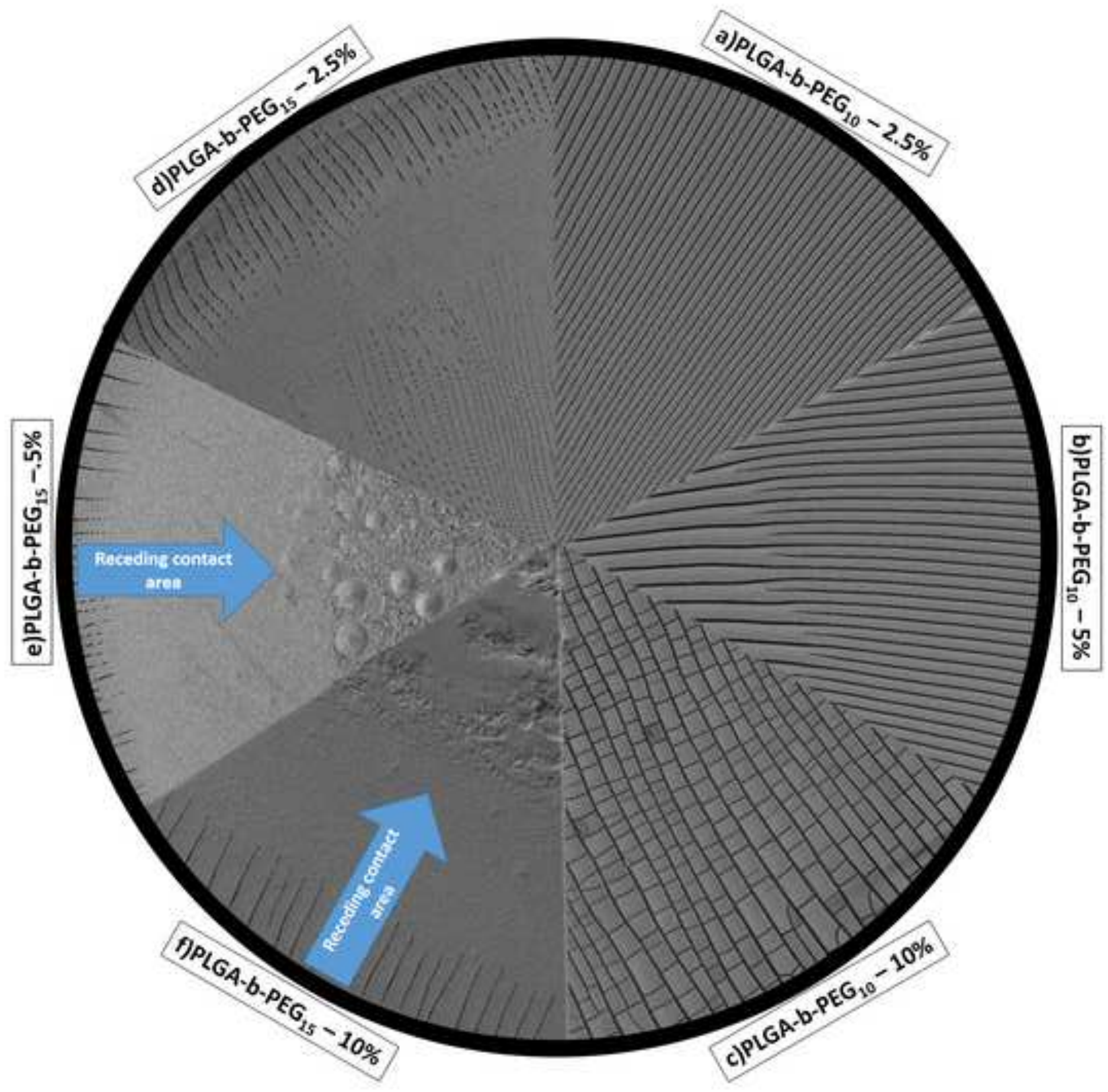


5: Figure
Click here to download high resolution image

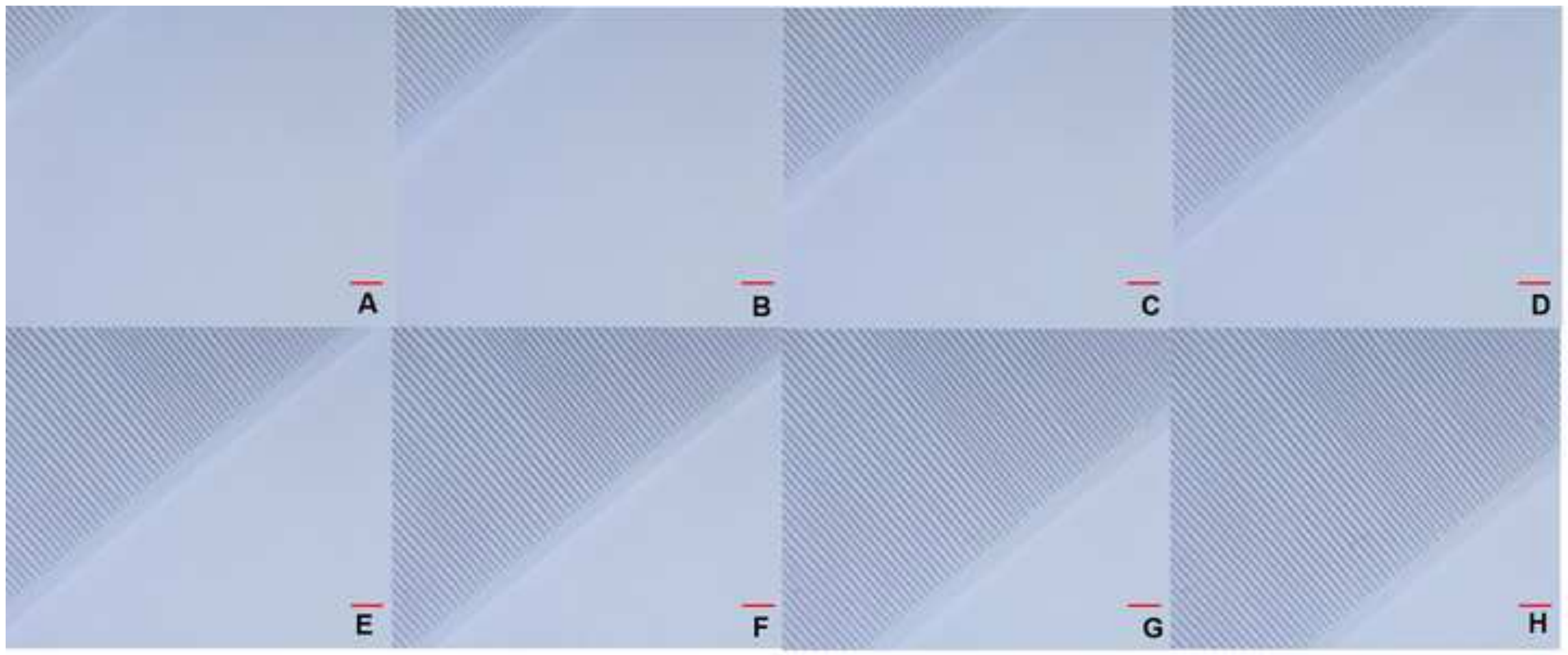


Table 1. The effect of PLGA-b-PEG10 (PEG10 indicates PEG content in 10\%) concentration and the water/oil flow ratio in the microfluidic device on nanoparticle size (DLS and SAXS), PDI and zeta potential.

\begin{tabular}{|c|c|c|c|c|c|c|}
\hline & $\begin{array}{l}\text { Polymer } \\
\%(w / v)\end{array}$ & $\begin{array}{l}\text { Water/oil } \\
\text { Flow } \\
\text { ratio }\end{array}$ & $\begin{array}{l}\text { Size } \\
\text { DLS } \\
(\mathrm{nm})\end{array}$ & $\begin{array}{l}\text { Poly dispersity } \\
\text { index (PDI) }\end{array}$ & $\begin{array}{c}\text { Zeta } \\
\text { Potential } \\
(\mathrm{mV})\end{array}$ & $\begin{array}{c}\text { Size } \\
\text { SAXS } \\
(\mathrm{nm})\end{array}$ \\
\hline \multirow{9}{*}{ 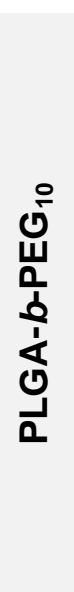 } & \multirow{3}{*}{2.5} & 1 & $140 \pm 1$ & $0.14 \pm 0.2$ & $-12.2 \pm 0.2$ & 141 \\
\hline & & 2 & $141 \pm 1$ & $0.13 \pm 0.1$ & $-8.1 \pm 0.4$ & 112 \\
\hline & & 3 & $140 \pm 1$ & $0.12 \pm 0.1$ & $-7.5 \pm 0.2$ & 128 \\
\hline & \multirow{3}{*}{5} & 1 & $107 \pm 1$ & $0.06 \pm 0.01$ & $-8.5 \pm 0.4$ & 106 \\
\hline & & 2 & $107 \pm 1$ & $0.05 \pm 0.01$ & $-8.5 \pm 0.5$ & 107 \\
\hline & & 3 & $108 \pm 1$ & $0.04 \pm 0.02$ & $-6.4 \pm 0.7$ & 109 \\
\hline & \multirow{3}{*}{10} & 1 & $94 \pm 1$ & $0.15 \pm 0.2$ & $-7.2 \pm 0.3$ & 94 \\
\hline & & 2 & $102 \pm 1$ & $0.17 \pm 0.1$ & $-7.7 \pm 0.4$ & 94 \\
\hline & & 3 & $104 \pm 1$ & $0.16 \pm 0.1$ & $-6.3 \pm 0.7$ & 100 \\
\hline
\end{tabular}

Table 2. The effect of PEG content (15 in compare to 10 in Table 1) and polymer concentration on nanoparticles size, PDI and zeta potential.

$\begin{array}{cc}\underset{(w / v)}{P o l y m e r} \% & \text { Water/oil } \\ \text { Flow ratio }\end{array}$

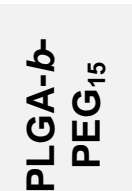

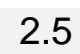

5

10

Size
DLS
(nm)

$175 \pm 1$

$203 \pm 1$

$266 \pm 2$

Poly dispersity
index (PDI)

$0.16 \pm 0.1$

$0.15 \pm 0.2$

$0.24 \pm 0.1$
Zeta

Potential

(mV)

$1266 \pm 2$

$-1.9 \pm 0.1$

$-1.5 \pm 0.1$

$-0.8 \pm 0.2$ 


\section{Declaration of interests}

$\bigotimes$ The authors declare that they have no known competing financial interests or personal relationships that could have appeared to influence the work reported in this paper.

$\square$ The authors declare the following financial interests/personal relationships which may be considered as potential competing interests:

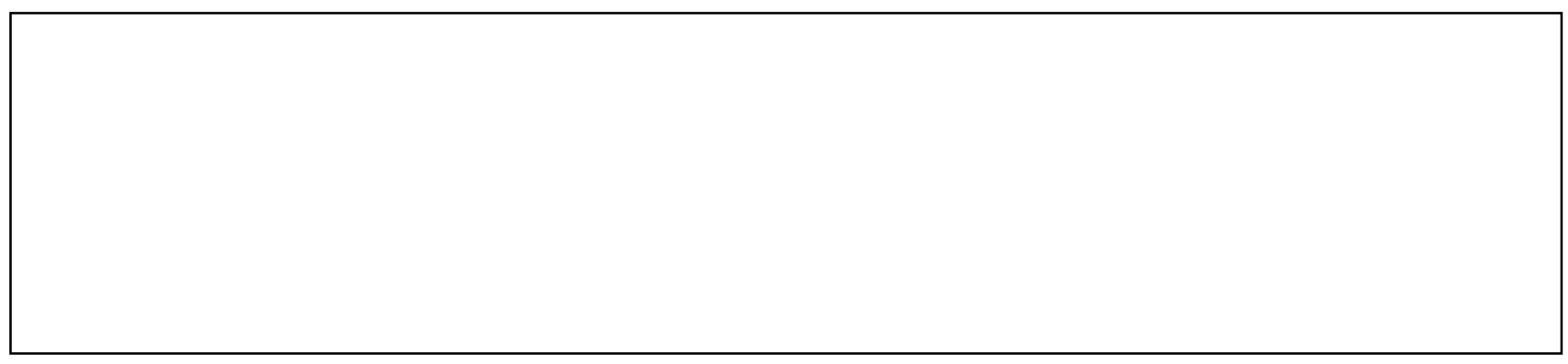

